\title{
\begin{tabular}{l|l} 
Mibraries & DSpace@MIT
\end{tabular}
}

\author{
MIT Open Access Articles
}

\section{A Certified Reduced Basis Method for the Fokker-Planck Equation of Dilute Polymeric Fluids: FENE Dumbbells in Extensional Flow}

The MIT Faculty has made this article openly available. Please share how this access benefits you. Your story matters.

Citation: Knezevic, David J., and Anthony T. Patera. “A Certified Reduced Basis Method for the Fokker-Planck Equation of Dilute Polymeric Fluids: FENE Dumbbells in Extensional Flow."

SIAM Journal on Scientific Computing 32.2 (2010): 793. (C) 2010 Society for Industrial and Applied Mathematics

As Published: http://dx.doi.org/10.1137/090759239

Publisher: Society for Industrial and Applied Mathematics (SIAM)

Persistent URL: http://hdl.handle.net/1721.1/61956

Version: Author's final manuscript: final author's manuscript post peer review, without publisher's formatting or copy editing

Terms of use: Creative Commons Attribution-Noncommercial-Share Alike 3.0 


\title{
A CERTIFIED REDUCED BASIS METHOD FOR THE FOKKER-PLANCK EQUATION OF DILUTE POLYMERIC FLUIDS: FENE DUMBBELLS IN EXTENSIONAL FLOW
}

\author{
DAVID J. KNEZEVIC* AND ANTHONY T. PATERA ${ }^{\dagger}$
}

\begin{abstract}
In this paper we present a reduced basis method for the parametrized FokkerPlanck equation associated with evolution of Finitely Extensible Nonlinear Elastic (FENE) dumbbells in a Newtonian solvent for a (prescribed) extensional macroscale flow. We apply a POD-Greedy sampling procedure for the stable identification of optimal reduced basis spaces and we develop a rigorous finite-time a posteriori bound for the error in the reduced basis prediction of the two outputs of interest - the optical anisotropy and the first normal stress difference. We present numerical results for stress-conformation hysteresis as a function of Weissenberg number and final which that demonstrate the rapid convergence of the reduced basis approximation and the effectiveness of the a posteriori error bounds.
\end{abstract}

Key words. Reduced basis methods, POD, greedy algorithm, a posteriori error estimation, Fokker-Planck equation, FENE dumbbells, polymeric fluids, micro-macro model.

1. Introduction. The flow of polymeric fluids can be described by a variety of mathematical models from purely phenomenological macroscale approaches to multiscale kinetic-theory based approaches $[8,9,37]$. The latter often take the form of the (Navier-)Stokes equation for the macroscale coupled to a Fokker-Planck description of the microscale: Stokes provides Fokker-Planck with strain rate; Fokker-Planck provides Stokes with an "extra polymeric stress" [9]. The microscale description includes both a spring-mass model and Brownian effects (reflected in the convective and diffusive components of the Fokker-Planck equation): the spring model will typically include $n_{\mathrm{s}}$ springs each described by a prescribed force law - most simply Hookean, but more realistically FENE (Finitely Extensible Nonlinear Elastic) [43].

There are two approaches to the numerical solution of the Stokes Fokker-Planck system: in the first approach, the Fokker-Planck equation is replaced by the equivalent stochastic differential equations that are then treated by Monte Carlo techniques [36]; in the second approach, the Stokes Fokker-Planck system is retained in deterministic form and treated by numerical techniques for partial differential equations (PDEs) $[23,28,31]$. The first approach can more readily treat high-dimensional systems $\left(n_{\mathrm{s}}\right.$ large) but provides relatively slow convergence; variance reduction techniques $[10$, $11,26]$ can at least partially address the latter issue. The second approach can not readily treat high-dimensional systems but provides relatively faster convergence; recent "dimension-adaptive" proposals can at least partially address the former issue $[2,3]$. In this paper our interest is in the second, or "PDE," approach to the Stokes Fokker-Planck system. We restrict our attention here to the dumbbell model $\left(n_{s}=1\right)$ such that we can apply a standard finite element discretization; extension of our methodology to the $n_{s}>1$ case is a topic of interest for future work, as described briefly at the conclusion.

Even in the $n_{s}=1$ case the main difficulty with the PDE approach is dimensionality: in particular, the Fokker-Planck equation is posed over physical-configuration space - a $d \times d$ dimensional space. ${ }^{1}$ To simplify the problem physical and configura-

\footnotetext{
*Department of Mechanical Engineering, MIT (dknez@mit.edu), corresponding author.

${ }^{\dagger}$ Department of Mechanical Engineering, MIT (patera@mit.edu).

${ }^{1}$ Throughout this paper, $d$ refers both to the dimension of physical space and the dimension of configuration space for a single dumbbell, either 2 or 3 .
} 
tion space can be separated either by invoking an alternating direction method $[28,31]$ or by applying Lagrangian techniques to the physical-space convection term. The latter will be our point of departure here: the Fokker-Planck equation over the $d^{2}-$ dimensional space is replaced by $\mathcal{N}_{\text {phys }}$ Fokker-Planck equations over a $d$-dimensional configuration space; here $\mathcal{N}_{\text {phys }}$ is the number of nodal or quadrature points associated with, say, finite element discretization of the macroscale Stokes (plus extra stress) description in physical space. Hence we must solve many $\left(\mathcal{N}_{\text {phys }}\right)$ instantiations of a parametrized $d$-dimensional Fokker-Planck equation posed on configuration space; ${ }^{2}$ in this paper we develop a numerical scheme for the $d$-dimensional Fokker-Planck equation which is highly efficient in this many query context. We emphasize that we consider in this first paper only, and in isolation, the Fokker-Planck "building block" of the Stokes Fokker-Planck system; the full coupled problem will be addressed in future work.

We consider the application of a reduced basis method [1, 18, 35, 39] — more specifically the certified reduced basis method of $[15,32,33,40]$ - to the FENE Fokker-Planck equation. The reduced basis method addresses (rapid) evaluation of functional outputs of parametrized PDEs - in our case here, the strain rateparametrized Fokker-Planck system. The reduced basis approach accepts an expensive Offline stage in order to minimize in a subsequent Online stage the marginal cost of each new input parameter $\rightarrow$ output evaluation - in our case here, strain rate $\rightarrow$ extra stress evaluation. The reduced basis method is thus ideally suited for rapid evaluation in the many-query context - ultimately (in the coupled context) $\mathcal{N}_{\text {phys }}$ appeals to the Fokker-Planck system - as in this situation the cost of the Offline stage is asymptotically negligible. The reduced basis method is also well-suited to real-time or "deployed" contexts such as parameter estimation and control - for example, inference of constitutive constants [30] — as in this situation the cost of the Offline stage is deemed unimportant.

We shall consider the problem of a suspension of FENE dumbbells in an extensional flow: the flow is specified - which thus by executive fiat decouples the macroscale flow from Fokker-Planck (though not vice versa); furthermore, the strain rate is uniform over the physical domain - which eliminates (without appeal to Lagrangian techniques) the physical space coordinate from the Fokker-Planck equation. This simple extensional flow model problem is in fact of significant theoretical $[19,20,41]$ and experimental [38] interest and much studied in the context of stress-conformation hysteresis [16]. The outputs of interest are the optical anisotropy and first normal stress difference histories (quantities related to the macroscopic stress induced by an ensemble of polymers).

In Section 2 we describe the governing equations, the "truth" finite element discretization on which the reduced basis approximation is built, and finally the reduced basis (Galerkin) approximation. In Section 3 we present rigorous a posteriori bounds for the error in the reduced basis prediction relative to the truth finite element approximation; we develop estimators for the field variable in various norms as well as for the outputs of interest. We note that the application of model order reduction approaches to polymeric flows is not new $[2,3]$; our emphasis is on the development and application of rigorous a posteriori error bounds. In Section 4.1 we discuss the Construction-Evaluation procedure which ensures efficient identification of a reduced

\footnotetext{
${ }^{2}$ Each instantiation of the $d$-dimensional Fokker-Planck equation is parametrized by the macroscale strain rate - more precisely, Lagrangian macroscale strain rate history - associated with a nodal point in physical space, as well as (optionally) the FENE constitutive "constants".
} 
basis approximation space in the Offline stage and rapid (many-query) input-output evaluation in the Online stage; in Section 4.2 we summarize the POD-Greedy [22] procedure for construction of an "optimal" reduced basis approximation space. Finally, in Section 5 we present numerical results for the stress-conformation hysteresis problem: reduced basis convergence, error estimation effectivity, and computational efficiency.

2. Formulation and Discretization. We introduce the continuous formulation of the FENE Fokker-Planck equation in Section 2.1, the corresponding "truth" finite element discretization in Section 2.2, and finally the reduced basis approximation in Section 2.3.

2.1. Continuous Formulation. We shall non-dimensionalize our problem in terms of macroscale variables: $L$, the macro length scale, for all lengths; $U$, the macro velocity scale, for all velocities; and $L / U$ (the inverse macroscale strain rate) for all times. The Fokker-Planck equation for FENE dumbbells in a spatially uniform extensional flow is then given by

$$
\frac{\partial \psi}{\partial t}+\nabla_{q} \cdot\left(\underset{\approx}{\underset{\sim}{q}} \psi-\frac{1}{2 \mathrm{Wi}} \underset{\sim}{F}(\underset{\sim}{q}) \psi\right)=\frac{1}{2 \mathrm{Wi}} \Delta_{q} \psi
$$

Here $\psi$ is the probability density for the dumbbell configuration; $q$ is the configuration coordinate - end-to-end vector - in a configuration space $D ; D$ is a ball of radius $\sqrt{b}$, where $b$ is a non-dimensional measure of maximum dumbbell extension - typically in the range $10-1000 ; \nabla_{q}$ and $\Delta_{q}$ refer to the gradient and Laplacian operators with respect to the configuration coordinate; $t$ is time in the interval $(0, T]$; $\underset{\sim}{\kappa}$ is the (pure extensional) strain rate given by $\operatorname{diag}(1,-1) g(t)$, where $g(t)=1$ (respectively, 0) for $0<t \leq T / 2$ (respectively, $T / 2<t \leq T)^{3}$; Wi is the Weissenberg number $\mathrm{Wi}=U \lambda / L$, where $\lambda$ is the characteristic dumbbell relaxation time; and $\underset{\sim}{F}$ is the FENE force law, given by $\underset{\sim}{F}(\underset{\sim}{q})=\underset{\sim}{q} /\left(1-|\underset{\sim}{q}|^{2} / b\right)$. Our initial condition $\psi(q, t=0)=\psi_{0}(q)$ is taken to be the zero-strain-rate steady-state probability density, $\psi_{0} \equiv Z\left(1-|q|^{2} / b\right)^{b / 2}$, where $Z$ is a normalization constant chosen so that $\int_{D} \psi_{0}(q) \mathrm{d} q=1$. We impose a homogeneous Dirichlet boundary condition on $\psi$ over the boundãry of $D$; the FENE force law prevents dumbbells from extending to their maximum length. Note that since $\psi$ is a probability density it should remain nonnegative and furthermore satisfy $\int_{D} \psi(\underset{\sim}{q}, t) \mathrm{d} \underset{\sim}{q}=1$ for all times $t \in[0, T]$.

The FENE force term in (2.1) acts as an unbounded convection coefficient. To ensure stability, Chauvière \& Lozinski $[13,14,31]$ propose a new dependent variable $\hat{\psi}$ given by

$$
\hat{\psi}(\underset{\sim}{q}, \cdot) \equiv \psi(\underset{\sim}{q}, \cdot) /\left(1-|\underset{\sim}{q}|^{2} / b\right)^{s},
$$

for a positive constant $s$; we choose here $s=1$. It shall also prove convenient in our parametric context to rescale time $t \rightarrow \hat{t}$ where $\hat{t}=t / T$; recall that $T$ is the final time. Substitution of these changes in dependent and independent variables yields

\footnotetext{
${ }^{3}$ In the case of the coupled Stokes Fokker-Planck system, the $d \times d$ tensor $\underset{\approx}{\kappa}$ is the gradient of the macroscopic velocity field - here we take $\underset{\approx}{\kappa}$ to be the velocity gradient tensor for an extensional flow. Note that due to the (assumed) incompressibility of the fluid, $\underset{\approx}{\kappa}$ must in general be a traceless tensor.
} 
the transformed problem (cf. (37) in [29])

$$
\begin{aligned}
\frac{1}{T} \frac{\partial \hat{\psi}}{\partial \hat{t}}-\frac{1}{2 \mathrm{Wi}} \Delta_{q} \hat{\psi}=\left(1-\frac{|q|^{2}}{b}\right)^{-1}\left[\frac{d}{2 \mathrm{Wi}}\left(1-\frac{2}{b}\right)+\frac{2}{b}\left(\underset{\sim}{q^{\mathrm{T}}} \underset{\approx}{\hat{\sim}} q\right)\right] \hat{\psi} \\
+\left[\frac{1}{2 \mathrm{Wi}}\left(1-\frac{4}{b}\right)\left(1-\frac{|q|^{2}}{b}\right)^{-1} \underset{\sim}{q}-\underset{\approx}{\hat{\kappa}} \underset{\sim}{q}\right] \cdot \nabla_{q} \hat{\psi},
\end{aligned}
$$

where $\underset{\approx}{\hat{\kappa}}=\operatorname{diag}(1,-1) \hat{g}(\hat{t})$ for $\hat{g}(\hat{t})=g(\hat{t} T)$. Our initial condition is now $\hat{\psi}_{0}(\underset{\sim}{q})=$ $\psi_{0}(\underset{\sim}{q}) /\left(1-|\underset{\sim}{q}|^{2} / b\right) \in X$. It is shown in [29] that for any $b \geq 4$ there exists a unique weak solution $\hat{\psi} \in \mathrm{H}_{0}^{1}(D)$ to $(2.3)$ (and therefore also a corresponding unique $\psi \in$ $\mathrm{H}_{0}^{1}(D)$ for $\left.(2.1)\right)$.

We now introduce a parametrization of this equation that is appropriate for the purposes of this paper. The two parameters of interest for this problem are the Weissenberg number, Wi, and the final time, $T-b$ is assumed to be fixed, and $\underset{\widetilde{\kappa}}{\hat{\kappa}}$ is determined by the extensional flow. Hence, we let $\mu \equiv(\mathrm{Wi}, T) \in \mathcal{D}$, where $\mathcal{D}(\tilde{\mathrm{a}}$ bounded subset of $\mathbb{R}^{2}$ ) denotes the parameter domain of interest: we choose Wi $\in$ $\left[\mathrm{Wi}_{\min }, \mathrm{Wi}_{\max }\right]$ and $T \in\left[T_{\min }, T_{\max }\right]$ and hence $\mathcal{D}=\left[\mathrm{Wi}_{\min }, \mathrm{Wi}_{\max }\right] \times\left[T_{\min }, T_{\max }\right]$; we specify particular values later in the paper. Note that in the limit $\mathrm{Wi} \rightarrow 0, \psi$ departs negligibly from $\psi_{0}$; furthermore the time to reach steady state (i.e. the "diffusion time scale") is proportional to Wi. Hence to avoid "uninteresting" behavior we could consider a triangular parameter domain in which $T \leq C \mathrm{Wi}$ for some constant $C$; we prefer here the simpler approach of a rectangular domain $\mathcal{D}$ with a positive lower bound $\mathrm{Wi}_{\min }$.

We now introduce the weak formulation. Let $X \equiv \mathrm{H}_{0}^{1}(D) \equiv\left\{v \in \mathrm{L}^{2}(D)\right.$ : $\left.\nabla_{q} v \in \mathrm{L}^{2}(D),\left.v\right|_{\partial D}=0\right\}$, and equip this space with the inner product $(v, w)_{X} \equiv$ $\int_{D} \nabla_{q} v \cdot \nabla_{q} w \mathrm{~d} \underset{\sim}{q}$ and norm $\|v\|_{X} \equiv \sqrt{(v, v)_{X}}$. We also denote the $\mathrm{L}^{2}(D)$ inner product and norm by $(v, w) \equiv \int_{D} v w \mathrm{~d} q$ and $\|w\| \equiv \sqrt{(w, w)}$, respectively. Then, following [29], we consider the weak form of $(2.3)$ : for $\hat{t} \in(0,1]$, find $\hat{\psi}(\hat{t} ; \underset{\sim}{\mu}) \in X$ such that

$$
\frac{1}{T}\left(\frac{\partial \hat{\psi}(\underset{\sim}{\mu})}{\partial \hat{t}}, \varphi\right)+\frac{1}{2 \mathrm{Wi}} a(\hat{\psi}(\underset{\sim}{\mu}), \varphi)-c(\hat{\psi}(\underset{\sim}{\mu}), \varphi ; \hat{t} ; \underset{\sim}{\mu})-d(\hat{\psi}(\underset{\sim}{\mu}), \varphi ; \hat{t} ; \underset{\sim}{\mu})=0, \quad \forall \varphi \in X,
$$

where

$$
\begin{aligned}
a(v, w) & =\int_{D} \nabla_{q} v \cdot \nabla_{q} w \mathrm{~d} \underset{\sim}{q}, \\
c(v, w ; \hat{t} ; \underset{\sim}{\mu}) & \left.=\int_{D}\left(1-\frac{|q|^{2}}{b}\right)^{-1}\left[\frac{d}{2 \mathrm{Wi}}\left(1-\frac{2}{b}\right)+\underset{b}{2} \underset{\sim}{q^{\mathrm{T}}} \underset{\sim}{\hat{\kappa}} \underset{\sim}{q}\right)\right] v w \mathrm{~d} \underset{\sim}{q}, \\
d(v, w ; \hat{t} ; \underset{\sim}{\mu}) & =\int_{D}\left[\frac{1}{2 \mathrm{Wi}}\left(1-\frac{4}{b}\right)\left(1-\frac{|q|^{2}}{b}\right)^{-1} \underset{\sim}{q} \underset{\sim}{\hat{\kappa}} \underset{\sim}{q}\right] \cdot \nabla_{q} v w \mathrm{~d} \underset{\sim}{q},
\end{aligned}
$$

are continuous bilinear forms.

The output quantities of interest for relaxation of extensional flow are the first 
normal stress difference (N.S.)

$$
N . S .(\hat{t} ; \underset{\sim}{\mu})=l_{1}(\hat{\psi}(\hat{t} ; \underset{\sim}{\mu})) \equiv \int_{D}\left(q_{1}^{2}-q_{2}^{2}\right) \hat{\psi}(\underset{\sim}{q}, \hat{t} ; \underset{\sim}{\mu}) \underset{\sim}{q},
$$

and the optical anisotropy (O.A.),

$$
\left.O \cdot A .(\hat{t} ; \underset{\sim}{\mu})=l_{2}(\hat{\psi}(\hat{t} ; \underset{\sim}{\mu})) \equiv \int_{D}\left(1-\frac{|\underset{\sim}{q}|^{2}}{b}\right)\left(q_{1}^{2}-q_{2}^{2}\right) \hat{\psi} \underset{\sim}{\underset{\sim}{q}}, \underset{\sim}{\hat{t}} \underset{\sim}{\mu}\right) \mathrm{d} \underset{\sim}{q} .
$$

Both $l_{1}$ and $l_{2}$ are bounded linear functionals on $X$ and in fact bounded linear functionals over $\mathrm{L}^{2}(D)$ - as we shall exploit subsequently. The first normal stress difference is the difference between the $x x$ and $y y$ components of the polymeric extra stress tensor [9] and the optical anisotropy is an experimentally measurable quantity that gives insight into the anisotropy of dumbbell stretching.

REMARK 2.1. For the sake of concreteness, we have developed the formulation and parametrization above for the specific case of relaxation of extensional flow. However, it is worth emphasizing that the computational framework and a posteriori error analysis developed in the subsequent sections are valid for arbitrary $\underset{\approx}{\hat{\kappa}}(\hat{t})$ - not just $\operatorname{diag}(1,-1) \hat{g}(\hat{t})$. The $O\left(d^{2}\right)$ (here $d$ is the dimension of physical space) additional parameters can be readily treated by the reduced basis approach. Moreover, the framework developed here can also be adapted to the case in which $b$ is a parameter rather than a fixed constant.

REMARK 2.2. We set $d=2$ above for the planar extensional flow problem under consideration, but the computational approach developed here can also be applied for $d=3$ - we emphasize this point in the sequel by continuing to carry the variable $d$.

2.2. Truth discretization. We now develop an Euler-backward finite element discretization of (2.4) which shall henceforth be referred to as the "truth" formulation. We shall build the reduced basis approximation on this truth approximation, and we shall measure the error in the reduced basis approximation relative to this truth approximation.

Let $X^{\mathcal{N}} \subset X$ be a finite element space of dimension $\mathcal{N}$ and let $\hat{t}^{k}=k \Delta \hat{t}, k=$ $0, \ldots, K$, for $\Delta \hat{t}=1 / K$. For a given $b \geq 4$, find $\hat{\psi}^{\mathcal{N} k}(\underset{\sim}{\mu}) \equiv \hat{\psi}^{\mathcal{N}}\left(\hat{t}^{k} ; \underset{\sim}{\mu}\right) \in X^{\mathcal{N}}$, $1 \leq k \leq K$, such that

$$
\begin{aligned}
& \frac{1}{T \Delta \hat{t}^{\prime}}\left(\hat{\psi}^{\mathcal{N} k}(\underset{\sim}{\mu})-\hat{\psi}^{\mathcal{N} k-1}(\underset{\sim}{\mu}), \varphi\right)+\frac{1}{2 \mathrm{Wi}} a\left(\hat{\psi}^{\mathcal{N} k}(\underset{\sim}{\mu}), \varphi\right) \\
& \quad-c\left(\hat{\psi}^{\mathcal{N} k}(\underset{\sim}{\mu}), \varphi ; \hat{t}^{k} ; \underset{\sim}{\mu}\right)-d\left(\hat{\psi}^{\mathcal{N} k}(\underset{\sim}{\mu}), \varphi ; \hat{t}^{k} ; \underset{\sim}{\mu}\right)=0, \quad \forall \varphi \in X^{\mathcal{N}} ;
\end{aligned}
$$

for our initial condition we take $\hat{\psi}^{\mathcal{N} 0}$ as the $\mathrm{L}^{2}(D)$ projection of $\hat{\psi}_{0}$ onto $X^{\mathcal{N}}$. We can then evaluate the associated truth outputs, N.S..$^{\mathcal{N}}$ and $O . A .^{\mathcal{N}}$,

$$
N \cdot S .^{\mathcal{N} k}(\underset{\sim}{\mu})=l_{1}\left(\hat{\psi}^{\mathcal{N} k}(\underset{\sim}{\mu})\right), \quad O \cdot A .^{\mathcal{N} k}(\underset{\sim}{\mu})=l_{2}\left(\hat{\psi}^{\mathcal{N} k}(\underset{\sim}{\mu})\right),
$$

for $0 \leq k \leq K$.

REMARK 2.3. By integrating (2.1) over $D$ it is easy to see that $\frac{\mathrm{d}}{\mathrm{d} t} \int_{D} \psi \mathrm{d} q=0$, as must be the case for a probability density. Analogously, assuming $1-|\underset{q}{q}|^{2} / b \in X^{\mathcal{N}}$ and setting $\varphi=1-|\underset{\sim}{q}|^{2} / b$ in $(2.10)$, we obtain

$$
\begin{aligned}
\int_{D} \psi^{\mathcal{N} k}(\underset{\sim}{\mu}) \mathrm{d} \underset{\sim}{q}=\int_{D}\left(1-|\underset{\sim}{q}|^{2} / b\right) \hat{\psi}^{\mathcal{N} k}(\underset{\sim}{\mu}) \mathrm{d} \underset{\sim}{q} \\
=\int_{D}\left(1-|\underset{\sim}{q}|^{2} / b\right) \hat{\psi}^{\mathcal{N} k-1}(\underset{\sim}{\mu}) \mathrm{d} \underset{\sim}{q}=\int_{D} \psi^{\mathcal{N} k-1}(\underset{\sim}{\mu}) \mathrm{d} \underset{\sim}{q} .
\end{aligned}
$$


Hence, if $1-|q|^{2} / b \in X^{\mathcal{N}}$, the normalization condition $\int_{D} \psi_{0} \mathrm{~d} q=1$ will be conserved by the truth formulation for $1 \leq k \leq K$ (an analogous result is provided by Chauvière $\&$ Lozinski for their spectral method [14]). Note, however, that in practice we consider a numerical quadrature which introduces (typically small) error into this conservation property.

A second property of a probability density is non-negativity. Our scheme cannot guarantee non-negativity of $\psi$, but in our experience non-negativity is only violated by very under-resolved (truth or reduced basis) solutions that are in any event not of practical interest.

2.3. Reduced basis approximation. The reduced basis approximation now follows directly from (2.10). We suppose that we have generated a set of mutually $(\cdot, \cdot)_{X}$-orthonormal basis functions, $\left\{\xi_{n} \in X^{\mathcal{N}}, 1 \leq n \leq N_{\max }\right\}$ in terms of which we construct the $N_{\max }$ hierarchical reduced basis spaces $X_{N} \equiv \operatorname{span}\left\{\xi_{n}, 1 \leq n \leq N\right\}$, $1 \leq N \leq N_{\max }$. (A POD-Greedy algorithm for constructing the basis functions $\xi_{n}$ is detailed in Section 4.2.)

Then, given $\underset{\sim}{\mu} \in \mathcal{D}$, the reduced basis solution, $\left.\hat{\psi}_{N}^{k} \underset{\sim}{\mu}\right) \in X_{N}, 1 \leq k \leq K$, satisfies

$$
\begin{aligned}
& \frac{1}{T \Delta \hat{t}}\left(\hat{\psi}_{N}^{k}(\underset{\sim}{\mu})-\hat{\psi}_{N}^{k-1}(\underset{\sim}{\mu}), \varphi\right)+\frac{1}{2 \mathrm{Wi}} a\left(\hat{\psi}_{N}^{k}(\underset{\sim}{\mu}), \varphi\right) \\
& \quad-c\left(\hat{\psi}_{N}^{k}(\underset{\sim}{\mu}), \varphi ; \hat{t}^{k} ; \underset{\sim}{\mu}\right)-d\left(\hat{\psi}_{N}^{k}(\underset{\sim}{\mu}), \varphi ; \hat{t}^{k} ; \underset{\sim}{\mu}\right)=0, \quad \forall \varphi \in X_{N} ;
\end{aligned}
$$

for our initial condition we take $\hat{\psi}_{N}^{0}$ to be the $\mathrm{L}^{2}(D)$ projection of $\hat{\psi}^{\mathcal{N} 0}$ onto $X_{N}$. The outputs $N . S ._{N}, O . A \cdot_{N}$ are evaluated as

$$
N . S \cdot \underset{N}{k}(\underset{\sim}{\mu})=l_{1}\left(\hat{\psi}_{N}^{k}(\underset{\sim}{\mu})\right), \quad O . A \cdot{ }_{N}^{k}(\underset{\sim}{\mu})=l_{2}\left(\hat{\psi}_{N}^{k}(\underset{\sim}{\mu})\right),
$$

for $0 \leq k \leq K$.

REMARK 2.4. If $1-|q|_{\sim}^{2} / b \in X_{N}$, then the reduced basis method satisfies the same integral conservation property as the truth formulation (cf. Remark 2.3). Note that we do not explicitly enforce this inclusion, since the function $1-|q|^{2} / b$ is typically very well approximated by $X_{N}$ and hence the error introduced into the conservation statement is small.

3. A posteriori error bounds. In this section we derive error bounds for the reduced basis approximation relative to the truth formulation. We first develop in Section 3.1 some preliminary results; in Section 3.2, we derive an $\mathrm{L}^{2}(D)$ bound for $\hat{\psi}_{N}$, which also yields error bounds for the outputs N.S..$_{N}$ and $O . A ._{N}$; we next derive in Section 3.3 an a posteriori error bound in the "energy" norm; finally, in Section 3.4, we discuss a technique for computing the stability factor lower bounds that arise in the error estimates.

3.1. Preliminaries. Let $\left.e_{N}^{k} \underset{\sim}{\mu}\right) \in X^{\mathcal{N}}$ denote the error in the reduced basis solution with respect to the truth solution, $\left.e_{N}^{k} \underset{\sim}{\mu}\right) \equiv \hat{\psi}^{\mathcal{N} k}(\underset{\sim}{\mu})-\hat{\psi}_{N}^{k}(\underset{\sim}{k})$. Also, let $r_{N}^{k}(\varphi ; \underset{\sim}{\mu})$ denote the residual

$$
\begin{aligned}
r_{N}^{k}(\varphi ; \underset{\sim}{\mu}) \equiv- & \frac{1}{T \Delta \hat{t}}\left(\hat{\psi}_{N}^{k}(\underset{\sim}{\mu})-\hat{\psi}_{N}^{k-1}(\underset{\sim}{\mu}), \varphi\right)-\frac{1}{2 \mathrm{Wi}} a\left(\hat{\psi}_{N}^{k}(\underset{\sim}{\mu}), \varphi\right) \\
& +c\left(\hat{\psi}_{N}^{k}(\underset{\sim}{\mu}), \varphi ; \hat{t}^{k} ; \underset{\sim}{\mu}\right)+d\left(\hat{\psi}_{N}^{k}(\underset{\sim}{\mu}), \varphi ; \hat{t}^{k} ; \underset{\sim}{\mu}\right), \quad \forall \varphi \in X^{\mathcal{N}},
\end{aligned}
$$


and define $\varepsilon_{N}^{k}(\underset{\sim}{\mu}), 1 \leq k \leq K$, to be the dual norm of the residual

$$
\varepsilon_{N}^{k}(\underset{\sim}{\mu})=\sup _{w \in X^{\mathcal{N}}} \frac{r_{N}^{k}(w ; \underset{\sim}{\mu})}{\|w\|_{X}}
$$

Finally, let $\hat{e}_{N}^{k}(\mu) \in X^{\mathcal{N}}$ denote the Riesz representation of the residual,

$$
\left(\hat{e}_{N}^{k}(\underset{\sim}{\mu}), v\right)=r_{N}^{k}(v ; \underset{\sim}{\mu}), \quad \forall v \in X^{\mathcal{N}},
$$

such that $\varepsilon_{N}^{k}(\mu)^{2}=\left\|\hat{e}_{N}^{k}(\mu)\right\|_{X}^{2}, 1 \leq k \leq K$. The latter result serves in the evaluation of our error bounds.

We shall also require the following stability factor in the a posteriori error bounds derived in Section 3.2 and 3.3,

$$
\sigma(\hat{t} ; \underset{\sim}{\mu})=\inf _{w \in X^{\mathcal{N}}} \frac{\|w\|_{X}^{2}+2 P(w, w ; \underset{\sim}{\mu})-Q(w, w ; \hat{t} ; \underset{\sim}{\mu})}{\|w\|^{2}}
$$

where

$$
\begin{gathered}
P(v, w) \equiv\left(1-\frac{4}{b}\right) \int_{D} \frac{|q|^{2}}{b}\left(1-\frac{|q|^{2}}{b}\right)^{-2} v w \mathrm{~d} \underset{\sim}{q}, \\
Q(v, w ; \hat{t} ; \underset{\sim}{\mu}) \equiv \int_{D}\left(d+\frac{8 \mathrm{~W} i}{b} \underset{\sim}{q^{\mathrm{T}}} \underset{\sim}{\hat{\kappa}}(\hat{t}) \underset{\sim}{q}\right)\left(1-\frac{|q|^{2}}{b}\right)^{-1} v w \mathrm{~d} \underset{\sim}{q},
\end{gathered}
$$

for $v, w \in X$. In fact, in practice we will evaluate a lower bound, $\sigma_{\mathrm{LB}}(\hat{t} ; \underset{\sim}{\mu})$, for $\sigma(\hat{t} ; \underset{\sim}{\mu})$ — we discuss a technique for efficiently constructing $\sigma_{\mathrm{LB}}(\hat{t} ; \mu)$ in Section 3.4. The next proposition asserts that the quotient in (3.4) is bounded below, and hence that $\sigma(\hat{t} ; \mu)$ is well-defined.

Proposition 3.1. Suppose that $b \geq 4$, then there exists a (possibly negative) constant $C \in \mathbb{R}$ which depends only on $b, d$, and Wi such that for all $w \in X$

$$
\frac{\|w\|_{X}^{2}+2 P(w, w)-Q(w, w ; \hat{t} ; \underset{\sim}{\mu})}{\|w\|^{2}} \geq C .
$$

Proof. The proof follows closely the argument from Section 3.2 of [29]. For any $\alpha>0$, we have

$$
\int_{D}\left(1-\frac{\left.|q|\right|^{2}}{b}\right)^{-1} w^{2} \mathrm{~d} \underset{\sim}{q} \leq \frac{1}{2 \alpha}(w, w)+\frac{\alpha}{2} \int_{D}\left(1-\frac{\left.|q|\right|^{2}}{b}\right)^{-2} w^{2} \mathrm{~d} \underset{\sim}{\sim},
$$

and by Hardy's inequality (see, for example, [6]) we further obtain

$$
\int_{D}\left(1-\frac{|q|^{2}}{b}\right)^{-2} w^{2} \mathrm{~d} q \leq 4 b\|w\|_{X}^{2}, \quad \forall w \in X .
$$

Therefore, setting $\alpha$ so that $2 b \alpha\left(d+8 \mathrm{Wi}\|\hat{\widetilde{\kappa}}(\hat{t})\|_{2}\right)=\frac{1}{2}$, where $\|\cdot\|_{2}$ denotes the matrix 2-norm, it follows (since $\underset{\sim}{q^{T}} \underset{\sim}{q}<b$ ) that

$$
\begin{aligned}
Q(w, w ; \hat{t} ; \underset{\sim}{\mu}) & \leq\left(d+8 \mathrm{Wi}\|\underset{\sim}{\hat{\sim}}(\hat{t})\|_{2}\right) \int_{D}\left(1-\frac{|q|^{2}}{b}\right)^{-1} w^{2} \mathrm{~d} \underset{\sim}{q} \\
& \leq 2 b\left(d+8 \mathrm{Wi}\|\underset{\sim}{\hat{\sim}}(\hat{t})\|_{2}\right)^{2}(w, w)+\frac{1}{2}\|w\|_{X}^{2} .
\end{aligned}
$$


Then,

$$
\begin{aligned}
\|w\|_{X}^{2}+2 P(w, w)-Q(w, w ; \hat{t} ; \underset{\sim}{\mu}) & \geq \frac{1}{2}\|w\|_{X}^{2}-2 b\left(d+8 \mathrm{Wi}\|\underset{\approx}{\hat{\kappa}}(\hat{t})\|_{2}\right)^{2}\|w\|^{2} \\
& \geq-2 b\left(d+8 \mathrm{Wi}\|\underset{\approx}{\hat{\sigma}}(\hat{t})\|_{2}\right)^{2}\|w\|^{2}
\end{aligned}
$$

for all $w \in X$; note $P(w, w) \geq 0$ since $b \geq 4$. Note also that, for our particular $\underset{\approx}{\hat{\kappa}}(\hat{t})$, $\|\underset{\approx}{\hat{\kappa}}(\hat{t})\|_{2}=1$ for $\hat{t} \in(0,1 / 2]$ and $\|\underset{\approx}{\hat{\kappa}}(\hat{t})\|_{2}=0$ for $\hat{t} \in(1 / 2,1]$.

3.2. $\mathrm{L}^{2}(D)$ and output error bounds. With the preceding definitions and with Proposition 3.1 in place, we can now prove Proposition 3.2, which gives a computable a posteriori error bound in the $\mathrm{L}^{2}(D)$ norm for the error $e_{N}^{k}(\underset{\sim}{\mu})[22]$.

Proposition 3.2. Suppose that $\Delta \hat{t}$ is sufficiently small so that

$$
\left(1+\frac{1}{2 \mathrm{Wi}} \sigma_{\mathrm{LB}}\left(\hat{t}^{k} ; \underset{\sim}{\mu}\right) T \Delta \hat{t}\right)>0, \quad 1 \leq k \leq K,
$$

and that $b \geq 4$ is fixed. Then for the reduced basis approximation defined in (2.13), we have

$$
\left\|e_{N}^{k}(\underset{\sim}{\mu})\right\| \leq \Delta_{N}^{k}(\underset{\sim}{\mu}), \quad 0 \leq k \leq K
$$

where

$$
\Delta_{N}^{k}(\underset{\sim}{\mu}) \equiv \sqrt{\frac{\left.\left\|e_{N}^{0}\right\|^{2}+2 \mathrm{Wi} T \Delta \hat{t} \sum_{j=1}^{k}\left(\varepsilon_{N}^{j} \underset{\sim}{\mu}\right)\right)^{2} \prod_{i=1}^{j-1}\left(1+\frac{1}{2 \mathrm{Wi}} \sigma_{\mathrm{LB}}\left(\hat{t}^{i} ; \underset{\sim}{\mu}\right) T \Delta \hat{t}\right)}{\prod_{i=1}^{k}\left(1+\frac{1}{2 \mathrm{Wi}} \sigma_{\mathrm{LB}}\left(\hat{t}^{i} ; \underset{\sim}{\mu}\right) T \Delta \hat{t}\right)}}
$$

is our $\mathrm{L}^{2}(D)$ error estimator.

Proof. It follows from (3.1) that

$$
\begin{aligned}
& \frac{1}{T \Delta \hat{t}}\left(e_{N}^{j}(\underset{\sim}{\mu})-e_{N}^{j-1}(\underset{\sim}{\mu}), \varphi\right)+\frac{1}{2 \mathrm{Wi}} a\left(e_{N}^{j}(\underset{\sim}{\mu}), \varphi\right) \\
& \left.\quad-c\left(e_{N}^{j}(\underset{\sim}{\mu}), \varphi ; \hat{t}^{j} ; \underset{\sim}{\mu}\right)-d\left(e_{N}^{j} \underset{\sim}{\mu}\right), \varphi ; \hat{t}^{j} ; \underset{\sim}{\mu}\right)=r_{N}^{j}(\varphi ; \underset{\sim}{\mu}), \quad \forall \varphi \in X^{\mathcal{N}} .
\end{aligned}
$$

Set $\left.\varphi=e_{N}^{j} \underset{\sim}{\mu}\right)$ to obtain

$$
\begin{aligned}
& \frac{1}{T \Delta \hat{t}}\left(e_{N}^{j}(\underset{\sim}{\mu})-e_{N}^{j-1}(\underset{\sim}{\mu}), e_{N}^{j}(\underset{\sim}{\mu})\right)+\frac{1}{2 \mathrm{Wi}}\left\|e_{N}^{j}(\underset{\sim}{\mu})\right\|_{X}^{2} \\
& \left.\quad-c\left(e_{N}^{j}(\underset{\sim}{\mu}), e_{N}^{j}(\underset{\sim}{\mu}) ; \hat{t}^{j} ; \underset{\sim}{\mu}\right)-d\left(e_{N}^{j} \underset{\sim}{\mu}\right), e_{N}^{j}(\underset{\sim}{\mu}) ; \hat{t}^{j} ; \underset{\sim}{\mu}\right)=r_{N}^{j}\left(e_{N}^{j}(\underset{\sim}{\mu}) ; \underset{\sim}{\mu}\right) .
\end{aligned}
$$

Using the identity $2(A-B) A=A^{2}-B^{2}+(A-B)^{2}$ on the first term in (3.13), we obtain the inequality

$$
\begin{gathered}
\left.\left\|e_{N}^{j}(\underset{\sim}{\mu})\right\|^{2}+\frac{T \Delta \hat{t}}{\mathrm{Wi}}\left\|e_{N}^{j}(\underset{\sim}{\mu})\right\|_{X}^{2} \leq\left\|e_{N}^{j-1}(\underset{\sim}{\mu})\right\|^{2}+2 T \Delta \hat{t} c\left(e_{N}^{j} \underset{\sim}{\mu}\right), e_{N}^{j}(\underset{\sim}{\mu}) ; \hat{t}^{j} ; \underset{\sim}{\mu}\right) \\
+2 T \Delta \hat{t} d\left(e_{N}^{j}(\underset{\sim}{\mu}), e_{N}^{j}(\underset{\sim}{\mu}) ; \hat{t}^{j} ; \underset{\sim}{\mu}\right)+2 T \Delta \hat{t} r_{N}^{j}\left(e_{N}^{j}(\underset{\sim}{\mu}) ; \underset{\sim}{\mu}\right),
\end{gathered}
$$

for $1 \leq j \leq K$ 
We now apply the identity $w \nabla_{q} w=\frac{1}{2} \nabla_{q}\left(w^{2}\right)$ and integrate by parts to find

$$
\begin{gathered}
d\left(w, w ; \hat{t}^{j} ; \underset{\sim}{\mu}\right)=\int_{D}\left[\frac{1}{2 \mathrm{Wi}}\left(1-\frac{4}{b}\right)\left(1-\frac{|q|^{2}}{b}\right)^{-1} \underset{\sim}{q}-\underset{\sim}{\hat{\kappa}}\left(\hat{t}^{j}\right) \underset{\sim}{q}\right] \cdot\left(\nabla_{q} w\right) w \mathrm{~d} \underset{\sim}{q} \\
=-\frac{1}{4 \mathrm{Wi}}\left(1-\frac{4}{b}\right) \int_{D} \nabla_{q} \cdot\left\{\left(1-\frac{|q|^{2}}{b}\right)^{-1} \underset{\sim}{q}\right\} w^{2} \mathrm{~d} \underset{\sim}{q}+\frac{1}{2} \int_{D} \nabla_{q} \cdot\left(\underset{\sim}{\hat{\sim}}\left(\hat{t}^{j}\right) \underset{\sim}{q}\right) w^{2} \mathrm{~d} \underset{\sim}{q} \\
=-\frac{1}{4 \mathrm{Wi}}\left(1-\frac{4}{b}\right) \int_{D}\left\{d\left(1-\frac{|\underset{\sim}{q}|^{2}}{b}\right)^{-1}+2 \frac{|\underset{\sim}{q}|^{2}}{b}\left(1-\frac{\mid \underset{\sim}{|q|^{2}}}{b}\right)^{-2}\right\} w^{2} \mathrm{~d} \underset{\sim}{q} \\
+\frac{1}{2} \int_{D} \nabla_{q} \cdot\left(\underset{\sim}{\hat{\kappa}}\left(\hat{t}^{j}\right) \underset{\sim}{q}\right) w^{2} \mathrm{~d} \underset{\sim}{q} .
\end{gathered}
$$

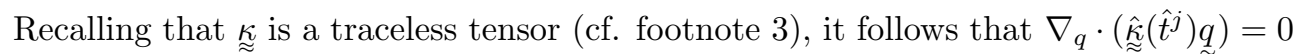
and hence

$$
d\left(w, w ; \hat{t}^{j} ; \underset{\sim}{\mu}\right)=-\frac{1}{4 \mathrm{Wi}}\left(1-\frac{4}{b}\right) \int_{D}\left\{d\left(1-\frac{|q|^{2}}{b}\right)^{-1}+2 \frac{|q|^{2}}{b}\left(1-\frac{|q|^{2}}{b}\right)^{-2}\right\} w^{2} \mathrm{~d} \underset{\sim}{ } .
$$

Hence

$$
\begin{aligned}
& c\left(w, w ; \hat{t}^{j} ; \underset{\sim}{\mu}\right)+d\left(w, w ; \hat{t}^{j} ; \underset{\sim}{\mu}\right)= \\
& -\frac{1}{2 \mathrm{Wi}}\left(1-\frac{4}{b}\right) \int_{D} \frac{|q|^{2}}{b}\left(1-\frac{|q|^{2}}{b}\right)^{-2} w^{2} \mathrm{~d} \underset{\sim}{q}+ \\
& \int_{D}\left(1-\frac{|q|^{2}}{b}\right)^{-1}\left[\frac{d}{2 \mathrm{Wi}}\left(1-\frac{2}{b}\right)+\frac{2}{b}\left(\underset{\sim}{q^{\mathrm{T}}} \underset{\approx}{\hat{\kappa}}\left(\hat{t}^{j}\right) \underset{\sim}{q}\right)-\frac{d}{4 \mathrm{Wi}}\left(1-\frac{4}{b}\right)\right] w^{2} \mathrm{~d} \underset{\sim}{q} \\
& =-\frac{1}{2 \mathrm{Wi}} P(w, w)+\int_{D}\left(1-\frac{|q|^{2}}{b}\right)^{-1}\left[\frac{d}{4 \mathrm{Wi}}+\frac{2}{b}\left({\underset{\sim}{q}}_{\sim}^{\mathrm{T}} \underset{\approx}{\hat{\kappa}}\left(\hat{t}^{j}\right) \underset{\sim}{q}\right)\right] w^{2} \mathrm{~d} \underset{\sim}{q} \\
& =-\frac{1}{2 \mathrm{Wi}} P(w, w)+\frac{1}{4 \mathrm{Wi}} Q\left(w, w ; \hat{t}^{j} ; \underset{\sim}{\mu}\right) \text {. }
\end{aligned}
$$

On substituting (3.16) into (3.14), we get

$$
\begin{aligned}
& \left.\left\|e_{N}^{j}(\underset{\sim}{\mu})\right\|^{2}+\frac{T \Delta \hat{t}}{\mathrm{Wi}}\left\|e_{N}^{j}(\underset{\sim}{\mu})\right\|_{X}^{2}+\frac{T \Delta \hat{t}}{\mathrm{Wi}} P\left(e_{N}^{j} \underset{\sim}{\mu}\right), e_{N}^{j}(\underset{\sim}{\mu})\right) \\
& \quad-\frac{T \Delta \hat{t}}{2 \mathrm{Wi}} Q\left(e_{N}^{j}(\underset{\sim}{\mu}), e_{N}^{j}(\underset{\sim}{\mu}) ; \hat{t}^{j} ; \underset{\sim}{\mu}\right) \leq\left\|e_{N}^{j-1}(\underset{\sim}{\mu})\right\|^{2}+2 T \Delta \hat{t} r_{N}^{j}\left(e_{N}^{j}(\underset{\sim}{\mu}) ; \underset{\sim}{\mu}\right),
\end{aligned}
$$

for $1 \leq j \leq K$.

We now apply the bound $r_{N}^{j}\left(e_{N}^{j}(\underset{\sim}{\mu}) ; \underset{\sim}{\mu}\right) \leq \varepsilon_{N}^{j}(\underset{\sim}{\mu})\left\|e_{N}^{j} \underset{\sim}{\mu}\right\|_{X}$ on the right-hand side of (3.17) and invoke Young's inequality to obtain

$$
\begin{gathered}
\left\|e_{N}^{j}(\underset{\sim}{\mu})\right\|^{2}+\frac{1}{2 \mathrm{Wi}} T \Delta \hat{t}\left\{\left\|e_{N}^{j}(\underset{\sim}{\mu})\right\|_{X}^{2}+2 P\left(e_{N}^{j}(\underset{\sim}{\mu}), e_{N}^{j}(\underset{\sim}{\mu})\right)-Q\left(e_{N}^{j}(\underset{\sim}{\mu}), e_{N}^{j}(\underset{\sim}{\mu}) ; \hat{t}^{j} ; \underset{\sim}{\mu}\right)\right\} \\
\leq\left\|e_{N}^{j-1}(\underset{\sim}{\mu})\right\|^{2}+2 \mathrm{Wi} T \Delta \hat{t}\left(\varepsilon_{N}^{j}(\underset{\sim}{\mu})\right)^{2},
\end{gathered}
$$


and hence,

$$
\left(1+\frac{1}{2 \mathrm{Wi}} \sigma_{\mathrm{LB}}\left(\hat{t}^{j} ; \underset{\sim}{\mu}\right) T \Delta \hat{t}\right)\left\|e_{N}^{j}(\underset{\sim}{\mu})\right\|^{2} \leq\left\|e_{N}^{j-1}(\underset{\sim}{\mu})\right\|^{2}+2 \mathrm{Wi} T \Delta \hat{t}\left(\varepsilon_{N}^{j}(\underset{\sim}{\mu})\right)^{2} .
$$

Recalling our hypothesis $\left(1+\frac{1}{2 \mathrm{Wi}} \sigma_{\mathrm{LB}}\left(\hat{t}^{i} ; \underset{\sim}{\mu}\right) T \Delta \hat{t}\right)>0$ for $1 \leq i \leq K$ and multiplying (3.18) through by $\prod_{i=1}^{j-1}\left(1+\frac{1}{2 \mathrm{Wi}} \sigma_{\mathrm{LB}}\left(\hat{t}^{i} ; \mu\right) \sim \sim \sim \hat{T}\right)$ gives

$$
\begin{gathered}
\left.\| e_{N}^{j} \underset{\sim}{\mu}\right)\left\|^{2} \prod_{i=1}^{j}\left(1+\frac{1}{2 \mathrm{Wi}} \sigma_{\mathrm{LB}}\left(\hat{t}^{i} ; \underset{\sim}{\mu}\right) T \Delta \hat{t}\right) \leq\right\| e_{N}^{j-1}(\underset{\sim}{\mu}) \|^{2} \prod_{i=1}^{j-1}\left(1+\frac{1}{2 \mathrm{Wi}} \sigma_{\mathrm{LB}}\left(\hat{t}^{i} ; \underset{\sim}{\mu}\right) T \Delta \hat{t}\right) \\
+2 \mathrm{Wi} T \Delta \hat{t}\left(\varepsilon_{N}^{j}(\underset{\sim}{\mu})\right)^{2} \prod_{i=1}^{j-1}\left(1+\frac{1}{2 \mathrm{Wi}} \sigma_{\mathrm{LB}}\left(\hat{t}^{i} ; \underset{\sim}{\mu}\right) T \Delta \hat{t}\right) .
\end{gathered}
$$

Summing from $j=1$ to $k$ gives

$$
\begin{aligned}
& \left\|e_{N}^{k}(\underset{\sim}{\mu})\right\|^{2} \prod_{i=1}^{k}\left(1+\frac{1}{2 \mathrm{Wi}} \sigma_{\mathrm{LB}}\left(\hat{t}^{i} ; \underset{\sim}{\mu}\right) T \Delta \hat{t}\right) \\
& \left.\quad \leq\left\|e_{N}^{0}\right\|^{2}+2 \mathrm{Wi} T \Delta \hat{t} \sum_{j=1}^{k}\left(\varepsilon_{N}^{j} \underset{\sim}{\mu}\right)\right)^{2} \prod_{i=1}^{j-1}\left(1+\frac{1}{2 \mathrm{Wi}} \sigma_{\mathrm{LB}}\left(\hat{t}^{i} ; \underset{\sim}{\mu}\right) T \Delta \hat{t}\right),
\end{aligned}
$$

which gives (3.10) for $1 \leq k \leq K$. Also, clearly $\Delta_{N}^{0}(\underset{\sim}{\mu})=\left\|e_{N}^{0}\right\|$, and hence the proof is complete.

It follows from Proposition 3.2 that we can develop a posteriori error bounds for the outputs N.S. and O.A. as provided by

Corollary 3.3. If the hypotheses of Proposition 3.2 are satisfied, then

$$
\begin{aligned}
& \left.\mid N . S .^{\mathcal{N} k}(\underset{\sim}{\mu})-N . S \cdot{ }_{N}^{k} \underset{\sim}{\mu}\right) \mid \leq \Delta_{N}^{N . S . k}(\underset{\sim}{\mu}), \\
& \left|O \cdot A \cdot{ }^{\mathcal{N} k}(\underset{\sim}{\mu})-O \cdot A_{N}^{k}(\underset{\sim}{\mu})\right| \leq \Delta_{N}^{O \cdot A \cdot k}(\underset{\sim}{\mu}),
\end{aligned}
$$

for $1 \leq k \leq K$, where

$$
\begin{aligned}
& \Delta_{N}^{N . S . k}(\underset{\sim}{\mu}) \equiv\left(\int_{D}\left(q_{1}^{2}-q_{2}^{2}\right)^{2} \mathrm{~d} q\right)^{\frac{1}{2}} \Delta_{N}^{k}(\underset{\sim}{\mu}), \\
& \Delta_{N}^{\text {O.A.k }}(\underset{\sim}{\mu}) \equiv\left(\int_{D}\left(1-\frac{|q|^{2}}{b}\right)^{2}\left(q_{1}^{2}-q_{2}^{2}\right)^{2} \mathrm{~d} \underset{\sim}{q}\right)^{\frac{1}{2}} \Delta_{N}^{k}(\underset{\sim}{\mu}),
\end{aligned}
$$

are our output error bounds.

Proof. For the N.S. $\underset{\sim}{\mu})$ error, we have from Cauchy-Schwarz and Proposition 3.2

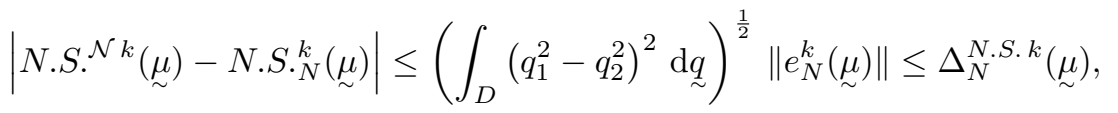

for $1 \leq k \leq K$. The bound for the $O . A$. output is derived analogously. 
3.3. Energy error bound. In this section we derive an a posteriori error bound in the "energy norm," $\|\cdot\|_{\ell^{2}\left(0, \hat{t}^{k} ; X\right)}$, which is defined as

$$
\|w\|_{\ell^{2}\left(0, \hat{t}^{k} ; X\right)}=\left(\frac{1}{k T \Delta \hat{t}} \sum_{j=1}^{k} T \Delta \hat{t}\left\|w\left(\hat{t}^{j}\right)\right\|_{X}^{2}\right)^{\frac{1}{2}}, \quad 1 \leq k \leq K .
$$

This energy norm bound is useful in the POD-Greedy algorithm developed in Section 4.2: we perform Greedy parameter selection based on the energy norm error bound (rather than the $\mathrm{L}^{2}(D)$ error bound) because this leads to greater consistency between the POD and Greedy components of the algorithm. The energy norm bound requires a second stability factor, $\tilde{\sigma}\left(\hat{t}^{k} ; \underset{\sim}{\mu}\right)$, defined as

$$
\tilde{\sigma}\left(\hat{t}^{k} ; \underset{\sim}{\mu}\right)=\inf _{w \in X^{\mathcal{N}}} \frac{\frac{1}{2}\|w\|_{X}^{2}+2 P(w, w)-Q\left(w, w ; \hat{t}^{k} ; \underset{\sim}{\mu}\right)}{\|w\|^{2}} .
$$

It follows from the same arguments as in the proof of Proposition 3.1 that $\tilde{\sigma}\left(\hat{t}^{k} ; \mu\right)$ is well-defined - bounded from below - for $b \geq 4$. Also, as in Section 3.1, we suppose that we are given a lower bound for $\tilde{\sigma}\left(\hat{t}^{k} ; \underset{\sim}{\mu}\right), \tilde{\sigma}_{\mathrm{LB}}\left(\hat{t}^{k} ; \underset{\sim}{\mu}\right)$. (The construction of these stability factor lower bounds is the topic of the next section.)

The energy norm bound is provided in

Proposition 3.4. Suppose that $\Delta \hat{t}$ is sufficiently small so that

$$
\left(1+\frac{1}{2 \mathrm{Wi}} \sigma_{\mathrm{LB}}\left(\hat{t}^{k} ; \underset{\sim}{\mu}\right) T \Delta \hat{t}\right)>0 \quad \text { and } \quad\left(1+\frac{1}{2 \mathrm{Wi}} \tilde{\sigma}_{\mathrm{LB}}\left(\hat{t}^{k} ; \underset{\sim}{\mu}\right) T \Delta \hat{t}\right)>0,
$$

for $1 \leq k \leq K$; further suppose that $b \geq 4$. For the reduced basis approximation defined in (2.13) we have

$$
\left\|e_{N}(\underset{\sim}{\mu})\right\|_{\ell^{2}\left(0, \hat{t}^{k} ; X\right)} \leq \Delta_{N}^{X k}(\underset{\sim}{\mu})
$$

for $1 \leq k \leq K$, where

$$
\begin{gathered}
\Delta_{N}^{X k}(\underset{\sim}{\mu}) \equiv\left\{\frac { 1 } { k T \Delta \hat { t } } \left(4 \mathrm{Wi}\left\|e_{N}^{0}\right\|^{2}-\sum_{\substack{j=1 \\
\tilde{\sigma}_{\mathrm{LB}}\left(\hat{t}^{j} ; \underset{\sim}{\mu}\right)<0}}^{k-1} 2 \tilde{\sigma}_{\mathrm{LB}}\left(\hat{t}^{j} ; \underset{\sim}{\mu}\right) T \Delta \hat{t}\left(\Delta_{N}^{j} \underset{\sim}{\mu}\right)^{2}\right.\right. \\
\left.\left.\left.+8 \mathrm{Wi}^{2} \sum_{j=1}^{k} T \Delta \hat{t}\left(\varepsilon_{N}^{j} \underset{\sim}{\mu}\right)\right)^{2}\right)\right\}^{\frac{1}{2}}
\end{gathered}
$$

is our energy norm error bound.

Proof. From (3.17), we have that

$$
\begin{aligned}
& \left\|e_{N}^{j}(\underset{\sim}{\mu})\right\|^{2}+\frac{1}{4 \mathrm{Wi}} T \Delta \hat{t}\left\|e_{N}^{j}(\underset{\sim}{\mu})\right\|_{X}^{2} \\
& +\frac{1}{2 \mathrm{Wi}} T \Delta \hat{t}\left\{\frac{1}{2}\left\|e_{N}^{j}(\underset{\sim}{\mu})\right\|_{X}^{2}+2 P\left(e_{N}^{j}(\underset{\sim}{\mu}), e_{N}^{j}(\underset{\sim}{\mu})\right)-Q\left(e_{N}^{j}(\underset{\sim}{\mu}), e_{N}^{j}(\underset{\sim}{\mu}) ; \hat{t}^{j} ; \underset{\sim}{\mu}\right)\right\} \\
& \quad \leq\left\|e_{N}^{j-1}(\underset{\sim}{\mu})\right\|^{2}+2 \mathrm{Wi} T \Delta \hat{t}\left(\varepsilon_{N}^{j}(\underset{\sim}{\mu})\right)^{2},
\end{aligned}
$$


and hence,

$$
\begin{aligned}
& \left(1+\frac{1}{2 \mathrm{Wi}} \tilde{\sigma}_{\mathrm{LB}}\left(\hat{t}^{j} ; \underset{\sim}{\mu}\right) T \Delta \hat{t}\right)\left\|e_{N}^{j}(\underset{\sim}{\mu})\right\|^{2}+\frac{1}{4 \mathrm{Wi}} T \Delta \hat{t}\left\|e_{N}^{j}(\underset{\sim}{\mu})\right\|_{X}^{2} \\
& \leq\left\|e_{N}^{j-1}(\underset{\sim}{\mu})\right\|^{2}+2 \mathrm{Wi} T \Delta \hat{t}\left(\varepsilon_{N}^{j}(\underset{\sim}{\mu})\right)^{2} .
\end{aligned}
$$

Therefore, it follows that

$$
\begin{aligned}
& \frac{1}{4 \mathrm{Wi}} T \Delta \hat{t}\left\|e_{N}^{j}(\underset{\sim}{\mu})\right\|_{X}^{2} \leq\left\|e_{N}^{j-1}(\underset{\sim}{\mu})\right\|^{2}-\left\|e_{N}^{j}(\underset{\sim}{\mu})\right\|^{2} \\
& \quad-\frac{1}{2 \mathrm{Wi}} \tilde{\sigma}_{\mathrm{LB}}\left(\hat{t}^{j} ; \underset{\sim}{\mu}\right) T \Delta \hat{t}\left\|e_{N}^{j}(\underset{\sim}{\mu})\right\|^{2}+2 \mathrm{Wi} T \Delta \hat{t}\left(\varepsilon_{N}^{j}(\underset{\sim}{\mu})\right)^{2} .
\end{aligned}
$$

Summing over $1 \leq j \leq k$ for any $1 \leq k \leq K$, we obtain

$$
\begin{gathered}
\sum_{j=1}^{k} T \Delta \hat{t}\left\|e_{N}^{j}(\underset{\sim}{\mu})\right\|_{X}^{2} \leq 4 \mathrm{Wi}\left\|e_{N}^{0}\right\|^{2}-4 \mathrm{Wi}\left(1+\frac{1}{2 \mathrm{Wi}} \tilde{\sigma}_{\mathrm{LB}}\left(\hat{t}^{k} ; \underset{\sim}{\mu}\right) T \Delta \hat{t}\right)\left\|e_{N}^{k}(\underset{\sim}{\mu})\right\|^{2} \\
\left.-\sum_{\substack{j=1 \\
\tilde{\sigma}_{\mathrm{LB}}\left(\hat{t}^{j} ; \underset{\sim}{\mu}\right)<0}}^{k-1} 2 \tilde{\sigma}_{\mathrm{LB}}\left(\hat{t}^{j} ; \underset{\sim}{\mu}\right) T \Delta \hat{t}\left(\Delta_{N}^{j}(\underset{\sim}{\mu})\right)^{2}+8 \mathrm{Wi}^{2} \sum_{j=1}^{k} T \Delta \hat{t}\left(\varepsilon_{N}^{j} \underset{\sim}{\mu}\right)\right)^{2} .
\end{gathered}
$$

By our hypothesis on $\Delta \hat{t}$, the last term on the first line in the previous inequality can be neglected; hence dividing through by $k T \Delta \hat{t}$ yields (3.27).

REMARK 3.5. We have derived error bounds specifically for the FENE FokkerPlanck equation, but in fact our arguments can be applied to any parabolic equation that satisfies a Gårding inequality - noting (3.16), it follows from Proposition 3.1 that (the elliptic part of the operator in) (2.10) satisfies a Gairding inequality, and indeed a Gärding inequality suffices to ensure boundedness of our stability constants. As a result, the methodology in this paper generalizes the "coercive" methodology in [32] to "non-coercive" parabolic equations.

3.4. Stability factor lower bound. In order to evaluate the stability factors $\sigma\left(\hat{t}^{k} ; \mu\right)$ or $\tilde{\sigma}\left(\hat{t}^{k} ; \mu\right)$ exactly we must find the minimum eigenvalue for the generalized eigenproblem posed on $X^{\mathcal{N}}$ corresponding to (3.4) or (3.26) — clearly a computationally intensive procedure. A more appropriate approach in the context of reduced basis methods is to compute rigorous lower bounds for the stability constants - the $\sigma_{\mathrm{LB}}\left(\hat{t}^{k} ; \underset{\sim}{\mu}\right)$ and $\tilde{\sigma}_{\mathrm{LB}}\left(\hat{t}^{k} ; \underset{\sim}{\mu}\right)$ that serve in Propositions 3.2 and 3.4. In general we can apply the Successive Constraint Method (SCM) [25] for this purpose; however, here a simpler technique suffices.

We first note that $\underset{\approx}{\hat{\kappa}}, \sigma$, and $\tilde{\sigma}$ can in fact be considered to be functions of $z \equiv$ $g(\hat{t}) \mathrm{Wi} \in\left[0, \mathrm{Wi}_{\max }\right]:$ we write $Q(w, w ; \hat{t} ; \underset{\sim}{\mu})=Q(w, w ; z)$ in $(3.4)$ and (3.26). Let $\left\{z_{i} \in\left[0, \mathrm{Wi}_{\max }\right], i=1, \ldots, n_{\sigma}\right\}$ be a set of points such that $z_{1}=0, z_{i+1}>z_{i}$, $z_{n_{\sigma}}=\mathrm{Wi}_{\max }$, and let $\mathcal{S} \equiv\left\{\left(z_{i}, \sigma\left(z_{i}\right)\right), i=1, \ldots, n_{\sigma}\right\}, \tilde{\mathcal{S}} \equiv\left\{\left(z_{i}, \tilde{\sigma}\left(z_{i}\right)\right), i=1, \ldots, n_{\sigma}\right\}$. We then define

$$
\sigma_{\mathrm{LB}}(z ; \mathcal{S}) \equiv \frac{z_{i+1}-z}{z_{i+1}-z_{i}} \sigma\left(z_{i}\right)+\frac{z-z_{i}}{z_{i+1}-z_{i}} \sigma\left(z_{i+1}\right), \quad \text { for } z \in\left[z_{i}, z_{i+1}\right],
$$

and

$$
\tilde{\sigma}_{\mathrm{LB}}(z ; \tilde{\mathcal{S}}) \equiv \frac{z_{i+1}-z}{z_{i+1}-z_{i}} \tilde{\sigma}\left(z_{i}\right)+\frac{z-z_{i}}{z_{i+1}-z_{i}} \tilde{\sigma}\left(z_{i+1}\right), \quad \text { for } z \in\left[z_{i}, z_{i+1}\right],
$$


corresponding to piecewise-linear approximations of $\sigma$ and $\tilde{\sigma}$, respectively.

We can then demonstrate

Proposition 3.6. Suppose that $b \geq 4$ and that the sets $\mathcal{S}$ and $\tilde{\mathcal{S}}$ are defined as above. Then $\sigma(z) \geq \sigma_{\mathrm{LB}}(z)$ and $\tilde{\sigma}(z) \geq \tilde{\sigma}_{\mathrm{LB}}(z)$ for any $z \in\left[0, \mathrm{Wi}_{\max }\right]$.

Proof. Let $i \in\left\{1, \ldots, n_{\sigma}-1\right\}$ be an index such that $z \in\left[z_{i}, z_{i+1}\right]$. Also, let $\theta \equiv\left(z_{i+1}-z\right) /\left(z_{i+1}-z_{i}\right) \in[0,1]$, so that $z=\theta z_{i}+(1-\theta) z_{i+1}$. Then we have

$$
\begin{aligned}
\sigma(z)= & \inf _{w \in X^{\mathcal{N}}} \frac{\|w\|_{X}^{2}+2 P(w, w)-Q(w, w ; z)}{\|w\|^{2}} \\
= & \inf _{w \in X^{\mathcal{N}}}\left\{\theta\left(\|w\|_{X}^{2}+2 P(w, w)-Q\left(w, w ; z_{i}\right)\right)\right. \\
& \left.\quad+(1-\theta)\left(\|w\|_{X}^{2}+2 P(w, w)-Q\left(w, w ; z_{i+1}\right)\right)\right\} /\|w\|^{2} \\
\geq \theta & \inf _{w \in X^{\mathcal{N}}} \frac{\|w\|_{X}^{2}+2 P(w, w)-Q\left(w, w ; z_{i}\right)}{\|w\|^{2}} \\
& \quad+(1-\theta) \inf _{w \in X^{\mathcal{N}}} \frac{\|w\|_{X}^{2}+2 P(w, w)-Q\left(w, w ; z_{i+1}\right)}{\|w\|^{2}} \\
=\theta & \sigma\left(z_{i}\right)+(1-\theta) \sigma\left(z_{i+1}\right)=\sigma_{\mathrm{LB}}(z) .
\end{aligned}
$$

The result for $\tilde{\sigma}_{\mathrm{LB}}$ is derived in the same way.

Assuming that $\sigma$ is a smooth function of $z$, we can estimate the error in this lower bound: for $z \in\left[z_{i}, z_{i+1}\right]$ we have (e.g. see Theorem 6.2 of [42])

$$
\left|\sigma(z)-\sigma_{L B}(z)\right| \leq C\left|z_{i+1}-z\right|\left|z-z_{i}\right|,
$$

where $C=\frac{1}{2} \max _{\zeta \in\left[z_{i}, z_{i+1}\right]}\left|\sigma^{\prime \prime}(\zeta)\right|-$ clearly the same argument applies to $\tilde{\sigma}$ as well. Note that in general a minimum eigenvalue (such as $\sigma$ or $\tilde{\sigma}$ ) will not be everywhere smooth with respect to parameter; however, smoothness is not required for Proposition 3.6, and (in the case of a discrete spectrum) (3.35) gives a useful insight into the accuracy of the lower bound method away from isolated points of discontinuity.

4. Computational procedures. In this section we focus on the efficient implementation of the reduced basis framework. In Section 4.1 we discuss the ConstructionEvaluation decomposition which enables rapid $-\mathcal{N}$-independent - evaluation of the output functionals and associated error bounds; in Section 4.2, we discuss a PODGreedy algorithm due to Haasdonk \& Ohlberger [22] for generating effective reduced basis spaces, $X_{N}$, such that $N \ll \mathcal{N}$.

4.1. Construction-evaluation decomposition. The calculation of the reduced basis outputs N.S. ${ }_{N}^{k}(\mu)$ and $O . A ._{N}^{k}(\mu)$, and the error bounds of Proposition 3.2, Corollary 3.3, and Proposition 3.4, admit a Construction-Evaluation decomposition. The expensive $-\mathcal{N}$-dependent - Construction stage, performed once, enables the subsequent very inexpensive $-\mathcal{N}$-independent - Evaluation stage, performed many times for each new desired $\mu \in \mathcal{D}$. As motivated in the Introduction, it is natural to pose the Fokker-Planck equation for polymeric fluids as a many-query problem: in this context the Construction-Evaluation decomposition leads to a highly efficient numerical method; the relevant computational cost metric is the asymptotic average cost - equivalently the cost of the (inexpensive) Evaluation stage.

The Construction-Evaluation decomposition must be applied (i) to obtain the reduced basis solution and in particular reduced basis outputs, (ii) to obtain the associated a posteriori error bounds as described by Proposition 3.2, Corollary 3.3, 
and Proposition 3.4, and (iii) to evaluate the stability constant lower bounds of Section 3.4. The key prerequisite is that the weak statement is "affine in parameter" $[32,40]$ : we define this requirement more precisely below, and we further demonstrate that our Fokker-Planck equation satisfies this crucial computational hypothesis. In fact, (i) and (ii) are already well treated in earlier papers on reduced basis approximation of parabolic PDEs $[32,34]$, and (iii) is a simpler version of the SCM procedure $[25,40]$. However, for completeness - and to give some sense of the fundamental ConstructionEvaluation notion - we include in the current paper a brief (problem-dependent) discussion of (i) and (iii). For convenience, we summarize the main results: the Evaluation operation count for (i) is $O\left(N^{3}+K N^{2}\right)$, for (ii) is $O\left(K N^{2}\right)$, and for (iii) is $O(1)$; hence the Evaluation cost is independent of $\mathcal{N}$ and in particular very rapid if $N \ll \mathcal{N}$.

To begin we note that (2.13) can be expressed as

$$
\begin{aligned}
\Theta_{1}(\underset{\sim}{\mu}) \beta_{1}\left(\hat{\psi}^{k}(\underset{\sim}{\mu}), \varphi\right)+\Theta_{2}(\underset{\sim}{\mu}) \beta_{2}\left(\hat{\psi}^{k}(\underset{\sim}{\mu}), \varphi\right) \\
\quad+\Theta_{3}\left(\hat{t}^{k}, \underset{\sim}{\mu}\right) \beta_{3}\left(\hat{\psi}^{k}(\underset{\sim}{\mu}), \varphi\right)=\Theta_{1}(\underset{\sim}{\mu}) \beta_{1}\left(\hat{\psi}^{k-1}(\underset{\sim}{\mu}), \varphi\right), \quad \forall \varphi \in X_{N},
\end{aligned}
$$

where,

$$
\begin{aligned}
\beta_{1}(v, w) \equiv & (v, w) \\
\beta_{2}(v, w) \equiv & \frac{1}{2} a(v, w)-\frac{1}{2} \int_{D}\left(1-\frac{4}{b}\right)\left(1-\frac{|q|^{2}}{b}\right)^{-1} \underset{\sim}{q} \cdot \nabla_{q} v w \mathrm{~d} \underset{\sim}{q} \\
& -\int_{D}\left(1-\frac{2}{b}\right)\left(1-\frac{|q|^{2}}{b}\right)^{-1} v w \mathrm{~d} \underset{\sim}{q}, \\
\beta_{3}(v, w) \equiv & \int_{D} \frac{2}{b}\left(q_{1}^{2}-q_{2}^{2}\right)\left(1-\frac{|q|^{2}}{b}\right)^{-1} v w \underset{\sim}{\mathrm{d}}-\int_{D}\left(q_{1} \frac{\partial v}{\partial q_{1}}-q_{2} \frac{\partial v}{\partial q_{2}}\right) w \mathrm{~d} q,
\end{aligned}
$$

and

$$
\Theta_{1}(\underset{\sim}{\mu})=\frac{1}{T \Delta \hat{t}}, \quad \Theta_{2}(\underset{\sim}{\mu})=\frac{1}{\mathrm{Wi}}, \quad \Theta_{3}(\hat{t}, \underset{\sim}{\mu})=-g(\hat{t}) .
$$

Recall $a(\cdot, \cdot)$ is defined in $(2.5)$. We say that our problem is affine in parameter (or more precisely, affine in functions of the parameter) because we can express the weak form as a sum of products of parameter- and time-dependent functions and parameter- and time- independent bilinear forms. In our case here, there are only three terms in this expansion. (Note that the $\Theta$ functions do not need to be affine functions of the parameter.)

REMARK 4.1. We emphasize that in fact the Fokker-Planck equation (2.13) is also affinely parametrizable in the case in which each of the components of $\underset{\widetilde{\sim}}{\hat{\kappa}} \in \mathbb{R}^{d \times d}$ is treated as an independent parameter. Furthermore, on rescaling each integral in (2.13) to the unit ball in $\mathbb{R}^{d}$, we can show that the Fokker-Planck equation remains affinely parametrizable in the case in which $b$ is included in the parametrization.

We first discuss the Construction-Evaluation approach for obtaining the outputs $N . S ._{N}^{k}\left(\underset{\sim}{)}\right.$ and $O . A ._{N}^{k}(\underset{\sim}{\mu})$. We suppose that $X_{N}=\operatorname{span}\left\{\xi_{j}, 1 \leq j \leq N\right\}$, and we expand $\hat{\psi}_{N}^{k}(\underset{\sim}{\mu}), 1 \leq k \leq K$, as $\left.\hat{\psi}_{N}^{k}(\underset{\sim}{\mu})=\sum_{j=1}^{N} \omega_{N j}^{k} \underset{\sim}{\mu}\right) \xi_{j}$. Upon insertion of this expansion into (4.1) we arrive at the discrete equations for $\omega_{N j}^{k}, 1 \leq j \leq N, 1 \leq k \leq$ 
$K$,

$$
\begin{gathered}
\sum_{j=1}^{N}\left(\Theta_{1}(\underset{\sim}{\mu}) \beta_{1}\left(\xi_{j}, \xi_{i}\right)+\Theta_{2}(\underset{\sim}{\mu}) \beta_{2}\left(\xi_{j}, \xi_{i}\right)+\Theta_{3}\left(\hat{t}^{k}, \underset{\sim}{\mu}\right) \beta_{3}\left(\xi_{j}, \xi_{i}\right)\right) \omega_{N j}^{k} \\
=\sum_{j=1}^{N} \Theta_{1}(\underset{\sim}{\mu}) \beta_{1}\left(\xi_{j}, \xi_{i}\right) \omega_{N j}^{k-1}, \quad 1 \leq i \leq N .
\end{gathered}
$$

Then, for example, N.S.N can be evaluated as $N . S_{.}^{k} \underset{\sim}{k}(\mu)=\sum_{j=1}^{N} \omega_{N j}^{k}(\underset{\sim}{\mu}) l_{1}\left(\xi_{j}\right)$. The Construction-Evaluation decomposition now follows readily.

In the Construction stage, we compute and store the parameter-independent $N \times N$ matrices corresponding to the bilinear forms $\beta_{q}\left(\xi_{j}, \xi_{i}\right), 1 \leq i, j \leq N, 1 \leq q \leq 3$, as well as the vectors $l_{i}\left(\xi_{j}\right), i \in\{1,2\}, 1 \leq j \leq N$. These calculations of course depend on $\mathcal{N}$. In the Evaluation stage, for a given $\mu \in \mathcal{D}$, and at each time level $k=1, \ldots, K$ : we first assemble the linear system (4.6) — we linearly combine the $N \times N$ matrices that were computed in the Construction stage; we then solve the resulting dense $N \times N$ linear system by LU-factorization; finally, we evaluate the output sums. The dominant computational cost in this Evaluation stage is the LU-factorization, which is $O\left(N^{3}\right)$; however, for our particular $g(\hat{t})$, we only need to perform this LU-factorization twice for a given $\mu$, and hence the total Evaluation cost $\underset{\sim}{\mu} \rightarrow N . S ._{N}^{k}(\mu), O . A ._{N}^{k}(\mu)$, $1 \leq k \leq K$, is $O\left(N^{3}+K N^{2}+N\right)$ operations (the back- and forward-substitutions at each time-step are $O\left(N^{2}\right)$ ).

Our a posteriori error bounds require Construction-Evaluation decompositions for the dual norm of the residual, $\varepsilon_{N}^{j}(\mu)$, and the stability factor lower bounds $\sigma_{\mathrm{LB}}(z)$ and $\tilde{\sigma}_{\mathrm{LB}}(z)$. The reader is referred to $[32,40]$ for the description of the ConstructionEvaluation procedure for the dual norm of the residual; we present here a simple Construction-Evaluation approach for the stability factor lower bounds of Section 3.4. In the Construction stage, we compute and store the sets $\mathcal{S}$ and $\tilde{\mathcal{S}}$ : we find the minimum eigenvalue of the generalized eigenproblems corresponding to (3.4) and (3.26) at each $z_{i}, i=1, \ldots, n_{\sigma}-$ a set of $2 n_{\sigma}$ expensive $\mathcal{N}$-dependent computations. In the Evaluation stage, for any $z \in\left[0, \mathrm{Wi}_{\max }\right]$, we first find the index $i$ such that $z \in\left[z_{i}, z_{i+1}\right]$; we then compute $\sigma_{\mathrm{LB}}(z)$ and $\tilde{\sigma}_{\mathrm{LB}}(z)$ as in (3.32), (3.33), which requires only $O(1)$ operations.

Before concluding the discussion of the Construction-Evaluation procedure, we wish to indicate the role of this computational decomposition within the context of the Offline and Online stages discussed in the Introduction. In the Offline stage we of course perform the Construction calculations, however, we also invoke Evaluation in efficiently building a good reduced basis space $X_{N}$ via the POD-Greedy process (cf. Section 4.2). In the Online stage - in which we wish to rapidly calculate, for any given new parameter, the outputs and associated output error bounds - we only invoke Evaluation computations; this leads to the low marginal computational cost that is desirable in the many-query and real-time contexts.

4.2. POD-Greedy sampling procedure. A crucial component of any reduced basis scheme is a method for constructing an appropriate space $X_{N}$. A Greedy training scheme for parabolic PDEs is proposed in [32] - this algorithm can yield good spaces, but it can also stall in many circumstances. The combination of a Proper Orthogonal Decomposition (POD) in time with a Greedy selection procedure in parameter is first proposed by Haasdonk \& Ohlberger [22] and overcomes the major shortcomings of 
the Greedy scheme by invoking the POD to properly account for temporal causality. In the present work, we directly use the POD-Greedy approach from [22], slightly modified (in norm) as described below.

Let $\operatorname{POD}\left(\left\{\hat{\psi}_{\mathcal{N}}^{k}(\mu), 0 \leq k \leq K\right\}, M\right)$ denote the $M$ largest POD modes - obtained via the "method of snapshots" — with respect to the $(\cdot, \cdot)_{X}$ inner product (see $[12,21]$ for more details): this operation returns an $(\cdot, \cdot)_{X}$ orthogonal set $\left\{\chi_{i}, 1 \leq i \leq M\right\}$ such that $\mathcal{P}_{M} \equiv \operatorname{span}\left\{\chi_{i}, 1 \leq i \leq M\right\}$ satisfies the optimality property

$$
\mathcal{P}_{M}=\arg \inf _{Y_{M} \subset \operatorname{span}\left\{\hat{\psi}_{\mathcal{N}}^{k}(\underset{\sim}{\mu}), 0 \leq k \leq K\right\}}\left(\frac{1}{K+1} \sum_{k=0}^{K} \inf _{v \in Y_{M}}\left\|\hat{\psi}_{\mathcal{N}}^{k}(\underset{\sim}{\mu})-v\right\|_{X}^{2}\right)^{1 / 2} .
$$

Here $Y_{M}$ denotes a linear space of dimension $M$. Note that the POD energy norm is closely related to the energy norm (3.25) except that the former contains a term corresponding to $k=0$ in the sum; it is for this reason that we prefer the energy norm (rather than $\mathrm{L}^{2}(D)$ norm) in the POD-Greedy procedure.

Let $\Xi_{\text {train }}$ denote a training set of $n_{\text {train }}$ (uniformly random) points in $\mathcal{D}$. Let $S^{*} \subset \mathcal{D}$ denote the set of greedily selected parameters; initialize $S^{*}=\left\{\mu_{0}^{*}\right\}$, where $\mu_{0}^{*} \in \mathcal{D}$ can be chosen arbitrarily. Specify $\Delta N$, the number of modes to be added to $\tilde{X}_{N}$ at each iteration: $\Delta N$ is typically chosen to be a small number, e.g. $\Delta N=1$; a larger $\Delta N$ will typically reduce Offline effort but increase Online effort - a less effective space. We also specify $N_{\max }$, the maximum dimension of our reduced basis spaces; in actual practice, we may replace $N_{\max }$ with an error tolerance termination criterion. Finally, let $e_{\text {proj }}^{k}(\underset{\sim}{\mu}) \equiv \hat{\psi}^{\mathcal{N} k}(\underset{\sim}{\mu})-\operatorname{proj}_{X_{N}} \hat{\psi}^{\mathcal{N} k}(\underset{\sim}{\mu})$, where $\operatorname{proj}_{X_{N}} \hat{\psi}^{\mathcal{N} k}(\underset{\sim}{\mu})$ is the $X$-orthogonal projection of $\hat{\psi}^{\mathcal{N} k}(\underset{\sim}{\mu})$ onto $X_{N}$. The POD-Greedy algorithm is then defined in Algorithm 1; this is essentially the same as the algorithm from [22] except that we use the energy norm bound in the Greedy parameter selection for greater consistency with the POD energy norm. Note that the set $E=\left\{e_{\text {proj }}^{k}\left(\mu_{\sim}^{*}\right), 0 \leq k \leq\right.$ $K\}$ is contained in the orthogonal complement of $X_{N}$, which naturally yields an orthogonal basis without further processing.

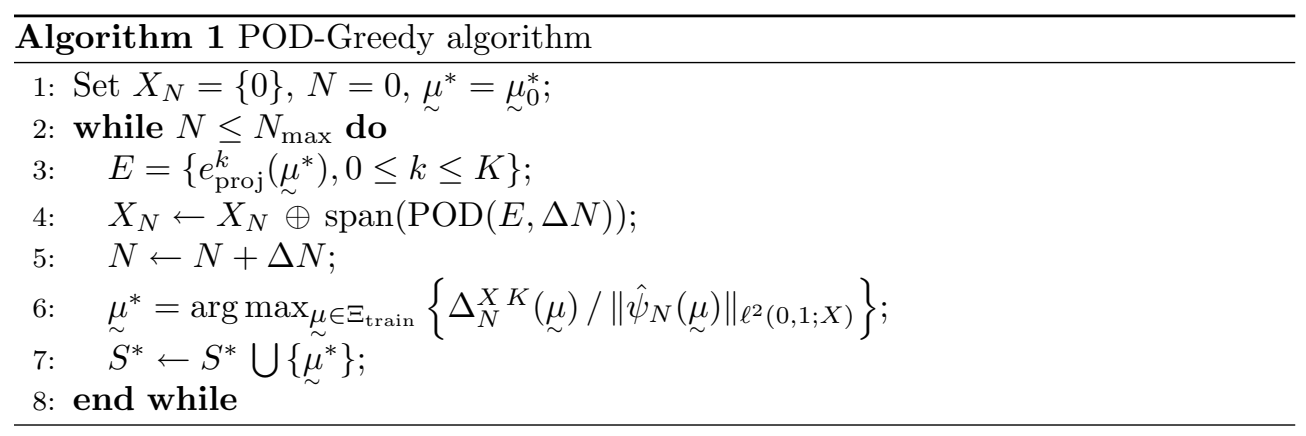

In principle, one can expect the POD-Greedy procedure discussed here to yield convergence down to machine precision - however, there is one important caveat that we must emphasize: the Construction-Evaluation decomposition introduces a plateau in the a posteriori error bounds (cf. Algorithm 1 line 6) at around square root of machine precision. ${ }^{4}$ Therefore, in Section 5 we shall compare two versions of the PODGreedy: the practical POD-Greedy, in which $\left.\Delta_{N}^{X}{ }^{K} \underset{\sim}{\mu}\right)$ is evaluated very inexpensively

\footnotetext{
${ }^{4}$ The Construction-Evaluation procedure for the dual norm of the residual calculates the very small quantity $\left(\varepsilon_{N}^{j}(\underset{\sim}{\mu})\right)^{2}$ as a sum of many $O(1)$ terms.
} 
(asymptotically) by the Construction-Evaluation decomposition, and the impractical POD-Greedy, in which we solve (3.3) directly - an $\mathcal{N}$-dependent operation - in order to compute $\Delta_{N}^{X} K(\underset{\sim}{K})$. The latter is solely of theoretical/pedagogical value.

5. Numerical results. In this section we present a series of computational results for the reduced basis framework applied to our extensional/relaxational flow problem: recall that the latter is defined by $(2.10)$ and parameters $\mu=(\mathrm{Wi}, T) \in$ $\mathcal{D} \equiv\left[\mathrm{Wi}_{\min }, \mathrm{Wi}_{\max }\right] \times\left[T_{\min }, T_{\max }\right]\left(\mathrm{Wi}_{\min }, T_{\min }>0\right)$; the outputs $N . \widetilde{S}$. and O.A. are defined in (2.11). Note that we set $b=10$ throughout this section. The reduced basis codes are based on the open source $\mathrm{C}++$ finite element library libMesh [27] which interfaces to PETSc [7] for numerical linear algebra functionality and to SLEPc [24] for eigenproblem routines.

We first present the parameter dependence of the field variable to illustrate the nature of the problem. We then present $\sigma_{\mathrm{LB}}\left(\tilde{\sigma}_{\mathrm{LB}}\right.$ is similar and hence omitted) to understand the behavior and implications of the stability factors. Next, we compare the practical and impractical POD-Greedy algorithms to demonstrate the numerical precision effect. Finally, we present reduced basis convergence plots and associated computational times to quantify the "reduced basis advantage," and we provide Online O.A.- N.S. hysteresis plots to demonstrate how our approach can (reliably) serve in practice in the many-query and real-time contexts. We conclude with a brief discussion of some challenges for future work.

We consider the truth finite element mesh shown in Figure 5.1(a): the space $X_{\mathcal{N}}$ is of dimension $\mathcal{N}=4261$; the mesh is graded to accurately resolve dumbbell extension along the $q_{1}$ axis. For the temporal discretization we choose $\Delta \hat{t}=0.01$ and hence $K=100$. We present plots of $\psi^{\mathcal{N}}$ at $\hat{t}=0,0.5,1$ for $\mathrm{Wi}=2.5$ and $T=4$ in Figures 5.1(b), (c), and (d), respectively. At $\hat{t}=0.5$, the dumbbells are highly stretched, resulting in sharp peaks in $\psi^{\mathcal{N}}$. The severity of the dumbbell extension is characterized by the Weissenberg number: Wi $<2.5$ (respectively, Wi $>2.5$ ) leads to less localized peaks (respectively, more localized peaks) than those shown in Figure 5.1(c). The challenge to the reduced basis method is hence twofold: to resolve the temporal variation due to the stretching and subsequent relaxation of the dumbbells, and to resolve the parametric dependence of the size, shape, and position of the probability density peaks.

With the aforementioned challenges in mind we now discuss the performance of the reduced basis method; we begin by examining the (lower bounds for the) stability constants. It is clear from the Propositions in Section 3 that the error bounds will be very sensitive to the "growth rate" $\sigma_{L B}$ (and $\tilde{\sigma}_{L B}$ ), as well as to the final time $T$ - roughly as $\exp \left(-\sigma_{\mathrm{LB}} / 2\right)$ for $\mathrm{Wi} \rightarrow 0, T \sim \mathrm{Wi}$, and $\operatorname{as} \exp \left(-\sigma_{\mathrm{LB}} T /(2 \mathrm{Wi})\right)$ for finite $\mathrm{Wi}, T$. In Figure 5.2 we plot $0.5 \sigma_{\mathrm{LB}}$ (relevant to small $\mathrm{Wi}$ ) for $z \in[0,1]$ and $0.5 \sigma_{\mathrm{LB}}(z) / z$ (relevant to finite $\mathrm{Wi}$ ) for $z \in[0.1,3]$; recall that we may interpret $z$ as $\mathrm{Wi}$ - more precisely, the magnitude of Wi $\|\underset{\sim}{\hat{\kappa}}(\hat{t})\|_{2}$. (We omit numerical results for $\tilde{\sigma}$ here with the understanding that it behaves analogously to $\sigma$.)

We make two observations based on Figure 5.2. First, unfortunately, $\sigma_{\mathrm{LB}}$ is negative for all $z$, and furthermore $\sigma_{\mathrm{LB}} / z$ is increasingly negative with increasing $z$ - the associated exponential growth will impose limitations on the range of Wi and $T$ for which we can obtain meaningful error bounds. (There is, however, no difficulty for small Wi: $\sigma_{\mathrm{LB}}$ tends to a constant as $z \rightarrow 0$.) Second, and more fortunately, we note that the exponential growth will be quite modest for $\mathrm{Wi} \sim O(1), T \sim O(1)-$ important elastic effects over macro timescales - and hence we can consider cases of physical interest; we demonstrate this point in our stress-conformation hysteresis 


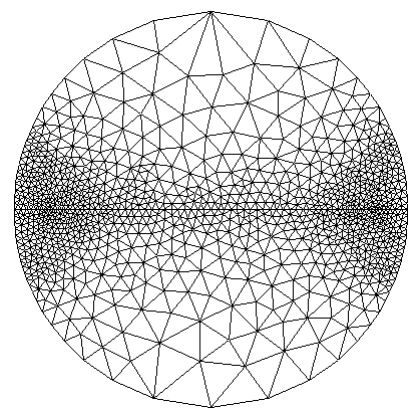

(a)

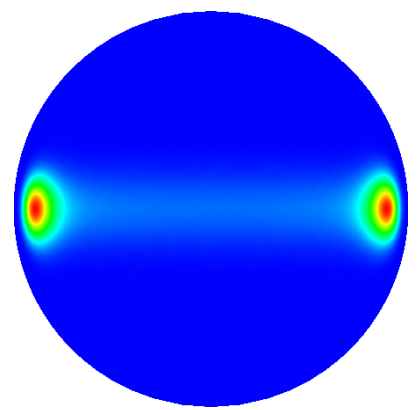

(c)

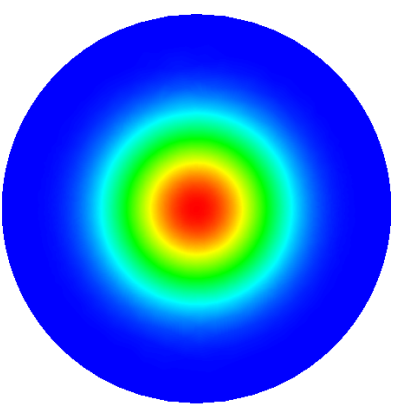

(b)

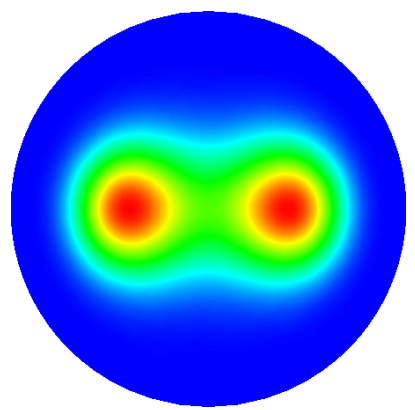

(d)

FIG. 5.1. (a) Truth finite element mesh; each second order isoparametric triangular element contains 6 nodes (also, although not shown in the figure, the domain boundary is interpolated quadratically). Plots (b), (c) and (d) show $\psi^{\mathcal{N}}$ for $\mathrm{Wi}=2.5, T=4$ at (b) $\hat{t}=0$ (blue: 0 , red: 0.19), (c) $\hat{t}=0.5$ (blue: 0, red: 0.75) and (d) $\hat{t}=1$ (blue: 0, red: 0.16).

results later in this section.

We now compare the behavior of the impractical and practical POD-Greedy methods. In order to demonstrate rapid convergence over many orders of magnitude we consider our stress relaxation extensional flow problem for the very modest parameter domain $\mathrm{Wi} \in[0.4,0.5], T \in[0.1,0.2]$. Let $E_{N}^{X, \max }$ denote the maximum relative energy error over a test set $\Xi_{\text {test }}$ of $n_{\text {test }}$ uniformly randomly generated points in $\mathcal{D}$,

$$
E_{N}^{X, \max } \equiv \max _{\underset{\sim}{\mu \in \Xi_{\text {test }}}}\left\{\left\|\hat{\psi}^{\mathcal{N}}(\underset{\sim}{\mu})-\hat{\psi}_{N}(\underset{\sim}{\mu})\right\|_{\ell^{2}(0,1 ; X)} /\left\|\hat{\psi}^{\mathcal{N}}(\underset{\sim}{\mu})\right\|_{\ell^{2}(0,1 ; X)}\right\}
$$

and let $\Delta_{N}^{X, \max }$ denote the corresponding relative error bound,

$$
\Delta_{N}^{X, \max } \equiv \max _{\sim}\left\{\Delta_{N}^{X} \Xi_{\text {test }}(\underset{\sim}{\mu}) /\left\|\hat{\psi}^{\mathcal{N}}(\underset{\sim}{\mu})\right\|_{\ell^{2}(0,1 ; X)}\right\} .
$$

We show in Figures 5.3 (a) and (b) the convergence of $E_{N}^{X, \max }$ and $\Delta_{N}^{X, \max }$ based on the impractical and practical versions of the POD-Greedy, respectively; all results in this section are for test samples of size $n_{\text {test }}=100$. We observe in Figure 5.3(a) that with the impractical POD-Greedy we are able to obtain convergence down to $O\left(10^{-11}\right)$ at $N=80$ - orders of magnitude below the plateau observed in Figure 5.3(b) for the practical POD-Greedy. Nevertheless, we shall henceforth exclusively use the practical POD-Greedy: in scientific applications we rarely require convergence below, say, 


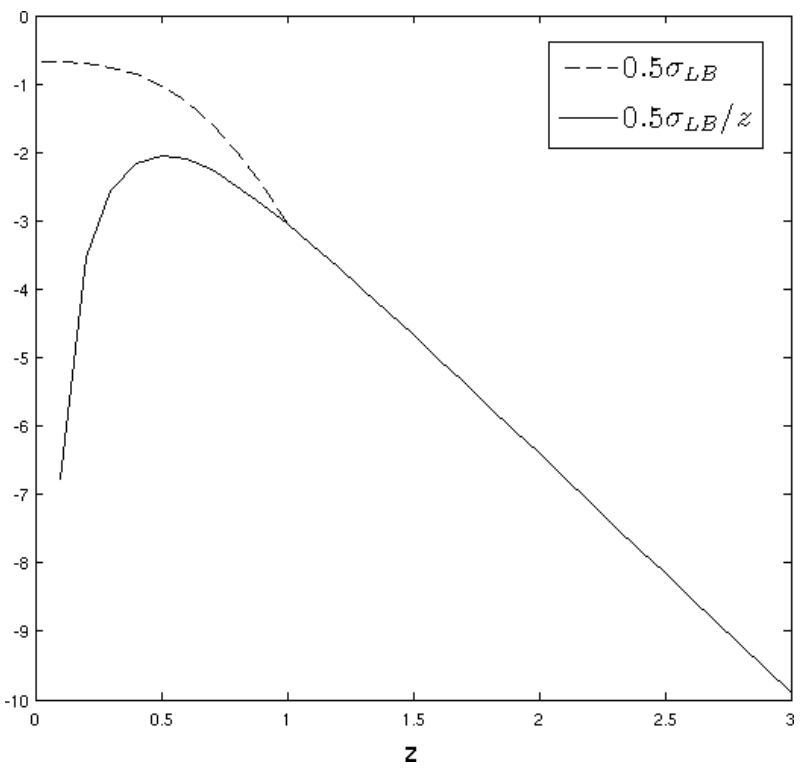

FIG. 5.2. Stability factor lower bound for $z \in[0,3]$ - the values of $\sigma_{L B}$ are obtained by the algorithm of Section 3.4 with $z_{i}=0.1 i, i=0, \ldots, 30$.

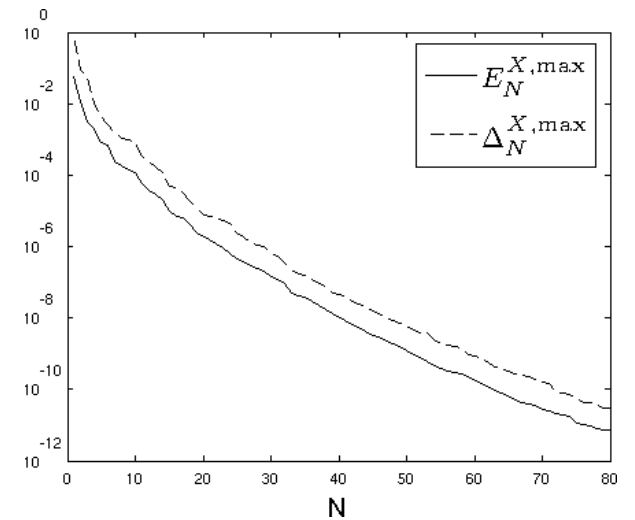

(a)

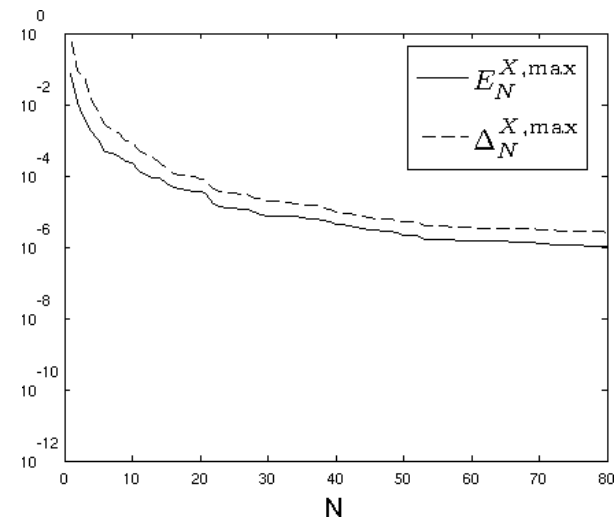

(b)

FIG. 5.3. Convergence of the reduced basis approximation for Wi $\in[0.4,0.5], T \in[0.1,0.2]$. The plots show convergence for (a) the impractical POD-Greedy - no error stagnation is observed, and (b) the practical POD-Greedy - error stagnation occurs at $O\left(10^{-6}\right)$ due to the ConstructionEvaluation decomposition for the error bounds.

$O\left(10^{-3}\right)$, and hence the error stagnation in Figure 5.3(b) will typically not be an issue in practice.

The practical (very inexpensive) POD-Greedy permits very extensive training samples $\Xi_{\text {train }}$ - we consider $n_{\text {train }}=400$ - and hence we can now apply our reduced basis framework in the remainder of this section to the much more interesting parameter domain $\mathcal{D}$ defined by $\mathrm{Wi}_{\min }=0.5, \mathrm{Wi}_{\max }=2.5, T_{\min }=1, T_{\max }=4$. We first consider the convergence of the reduced basis approximation for $\underset{\sim}{\mu} \in \mathcal{D}$ for $1 \leq N \leq 60=N_{\max }$ : we present in Figure 5.4(a) $E_{N}^{X, \max }$ and $\Delta_{N}^{X, \max }$; we present in 
Figure 5.4(b) the corresponding quantities for the output N.S., ${ }^{5}$

$$
\begin{aligned}
E_{N}^{N . S ., \max } & \equiv \max _{\sim}\left\{\in \Xi_{\text {test }}\left\{\left(\max _{0 \leq k \leq K} \mid N \cdot S .^{\mathcal{N} k}(\underset{\sim}{\mu})-N \cdot S_{N}^{k} \underset{\sim}{\mu}\right) \mid\right) /\left(\max _{0 \leq k \leq K}\left|N \cdot S^{\mathcal{N} k}(\underset{\sim}{\mu})\right|\right)\right\}, \\
\Delta_{N}^{N . S ., \max } & \equiv \max _{\sim}\left\{\Xi_{\text {test }}^{N . S . K}(\underset{\sim}{\mu}) /\left(\max _{0 \leq k \leq K}\left|N \cdot S .^{\mathcal{N} k}(\underset{\sim}{\mu})\right|\right)\right\} .
\end{aligned}
$$

We observe exponential convergence for the true error and the a posteriori error bound for both $\hat{\psi}_{N}$ and N.S.N (we omit O.A. - the convergence is roughly the same as for N.S.).

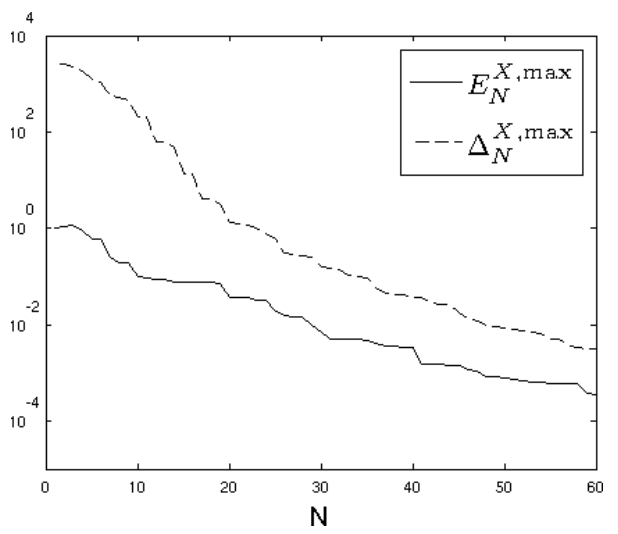

(a)

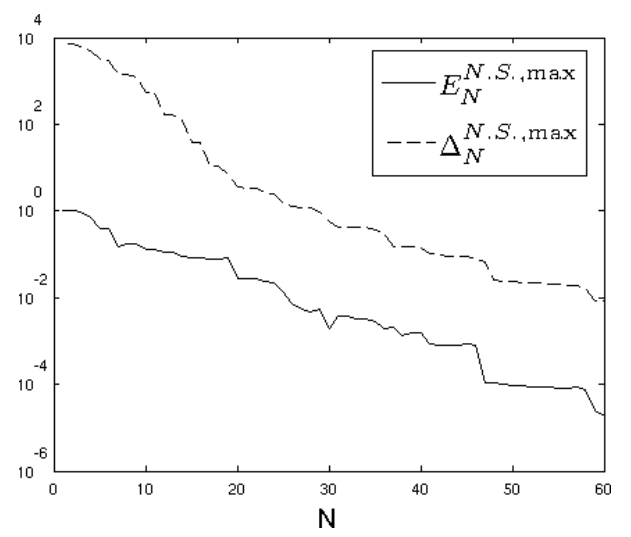

(b)

FIG. 5.4. Convergence of the reduced basis approximation for Wi $\in[0.5,2.5], T \in[1,4]$.

We present in Table 5.1 the speedup achieved by the reduced basis method: the speedup is defined as the truth computation time, $\underset{\sim}{\mu} \rightarrow N \cdot S^{\mathcal{N} k}(\underset{\sim}{\mu}), O \cdot A \cdot \cdot^{\mathcal{N} k}(\underset{\sim}{)})$, $1 \leq k \leq K$, divided by the Online reduced basis computation time, $\underset{\sim}{\mu} \rightarrow N . S{ }_{N}^{k}(\underset{\sim}{k})$, $\Delta_{N}^{N . S . k}(\underset{\sim}{\mu}), O \cdot A \cdot{ }_{N}^{k}(\underset{\sim}{\mu}), \Delta_{N}^{O . A . k}(\underset{\sim}{)}), 1 \leq k \leq K$; note that the Online time includes the calculation of both the outputs and the associated error bounds. (All times are averaged over our $\Xi_{\text {test }}$ sample.) We also include in the table the true output error and output error bounds. We observe, for example for $N=50$, that we obtain a speedup of over 100 for a maximum relative output error bound of less than $2.5 \%$. In short, in the Online stage, we can provide the accuracy and reliability of our expensive truth finite element calculation but at a small fraction of the cost.

Finally, we present numerical results for the outputs N.S. and O.A. and associated error bounds in a format of interest to experimentalists. In Figure 5.5, we show N.S. ${ }_{N}^{k}$ and N.S. ${ }_{N}^{k} \pm \Delta_{N}^{N . S . k}, 0 \leq k \leq K$, for different Wi and T. (Figure 5.5(a) illustrates the behavior alluded to in the discussion in Section 2.1 related to the time scaling as $\mathrm{Wi} \rightarrow 0$ : as we reduce $\mathrm{Wi}$, we deviate less from $\psi_{0}$ and we reach steady state more quickly in both the extensional and relaxational phases.) Figures 5.5(a) and (b) show that, as expected from stability and approximation arguments, fixed accuracy for higher $\mathrm{Wi}$ and $T$ requires larger $N$. Moreover, our error bounds, although not overly sharp (see Figure 5.4 and Table 5.1), are certainly meaningful: $N . S \cdot{ }_{N} \pm \Delta_{N}^{N}$.S.

\footnotetext{
${ }^{5}$ In actual Online practice, we would replace the denominator of $\Delta_{N}^{N . S ., \text { max }}$ with the inexpensive

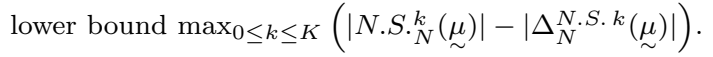




\begin{tabular}{|c|c|ccc|}
\hline$N$ & Online time $(\mathrm{s})$ & Speedup & $E_{N}^{N . S ., \max }$ & $\Delta_{N}^{N . S ., \max }$ \\
\hline 10 & $3.85 \mathrm{e}-3$ & $2.61 \mathrm{e}+3$ & $1.27 \mathrm{e}-1$ & $5.56 \mathrm{e}+2$ \\
20 & $1.91 \mathrm{e}-2$ & $5.27 \mathrm{e}+2$ & $2.65 \mathrm{e}-2$ & $3.59 \mathrm{e}+0$ \\
30 & $4.24 \mathrm{e}-2$ & $2.37 \mathrm{e}+2$ & $1.97 \mathrm{e}-3$ & $5.49 \mathrm{e}-1$ \\
40 & $6.57 \mathrm{e}-2$ & $1.53 \mathrm{e}+2$ & $1.54 \mathrm{e}-3$ & $1.44 \mathrm{e}-1$ \\
50 & $9.55 \mathrm{e}-2$ & $1.05 \mathrm{e}+2$ & $9.21 \mathrm{e}-5$ & $2.29 \mathrm{e}-2$ \\
60 & $1.30 \mathrm{e}-1$ & $7.74 \mathrm{e}+1$ & $1.79 \mathrm{e}-5$ & $8.28 \mathrm{e}-3$ \\
\hline
\end{tabular}

Online computation time, average speedup, relative N.S. error $\left(E_{N}^{N . S ., \max }\right)$ and relative N.S. error bound $\left(\Delta_{N}^{N . S ., \max }\right)$. The computation time for each truth solve is 10.1 seconds. All computations are performed on a $1.73 \mathrm{GHz}$ Intel processor.

is a very effective surrogate for N.S. ${ }^{\mathcal{N}}$. We also note that Figure 5.5(b) shows that FENE dumbbells respond differently to relaxation when the extensional flow has been imposed for different durations - for example, the initial relaxation transient (at $t=T / 2$ ) is steeper for $T=4$ than for $T=1$. This is a manifestion of the "memory" exhibited by dumbbells: the strain rate history from a time interval $[0, t)$ is in a sense encoded in the dumbbell configuration probability density at time $t$.

We demonstrate stress-conformation hysteresis in Figures 5.6 and 5.7 by plotting N.S. vs. O.A.; in these plots, the a posteriori error bounds are indicated as boxes each box defines a region in which the corresponding truth outputs (at the corresponding time point) must reside. Figure 5.6 shows hysteresis plots at different Weissenberg numbers, whereas Figure 5.7 demonstrates "convergence" of the error boxes - three plots for the same parameters, but with increasing $N$. The Online role of the error bounds both in confirming accuracy and in optimizing efficiency - by allowing us to certifiably satisfy a specified error tolerance (as indicated by the "tightness" of the error boxes) for $N<N_{\max }$ as small as possible - is clearly illustrated by Figure 5.7.

We next consider the impact of the effectivity of the error bounds: the effectivity is the ratio of the error bound to the true error. Clearly a large effectivity leads to extra work in both the Offline and Online stages: the Offline work is sensitively dependent on $N_{\max }$ (typically chosen to certifiably satisfy a given output error tolerance over $\left.\Xi_{\text {test }}\right)$; similarly, the Online speedup will be adversely affected by unnecessarily large $N$. Of particular concern is the effectivity of the error bound for the output, which is roughly an order of magnitude larger than the effectivity for the field variable - a consequence of the "worst case" Cauchy-Schwarz argument of Corollary 3.3. A topic of interest for future work is the development of a primal-dual approximation [32] to improve output effectivity - although it should be noted that this approach is less appealing in the case in which multiple outputs are of interest (cf. O.A. and N.S.).

To close, we briefly discuss three further challenges for future work. First, it is desirable to be able to treat $b$ significantly larger than 10, particularly if we wish to include $b$ in the parametrization of the problem (cf. Remark 4.1). ${ }^{6}$ The parameter $b$ is proportional to the FENE spring stiffness: a larger value of $b$ is more difficult from a reduced basis point of view - and indeed from a numerical point of view in general - because we obtain sharper and more localized solution profiles. A promising approach to address the sensitive parametric dependence for larger $b$ is a "multi-element" parameter domain decomposition $[4,5,17]$.

Second, as discussed in the Introduction, an important application of the reduced

\footnotetext{
${ }^{6} \mathrm{~A}$ reduced basis scheme with $b$ as a parameter could be used, for example, in a parameter identification study in which one seeks to deduce dumbbell parameters based on experimentally observed stress outputs.
} 
basis method developed here is the coupled Stokes Fokker-Planck system. Reduced basis treatment of the Stokes Fokker-Planck system would require (i) implementation of a Lagrangian "particle tracking" scheme for calculating strain rate histories, (ii)

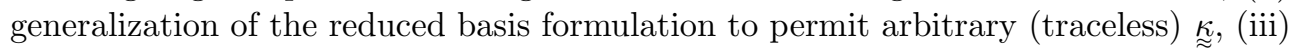
development of an appropriate scheme for construction of the reduced basis space in the case in which $\underset{\approx}{\approx}$ is an a priori unknown time-dependent tensor, and finally (iv) extension of our a posteriori results to the full coupled equations. (Note the resulting technology can also be applied to rod-like molecules for which $n_{s}=1$ in fact corresponds to physically relevant systems.)

Third, as discussed in the Introduction, a richer model of a polymer is obtained by using a chain of dumbbells $\left(n_{s}>1\right)$ rather than a single dumbbell. In this case the methodology developed in this paper is impracticable because the "truth" finite element solves would become intractable for $n_{s}>1$ due to the "curse of dimensionality." One appealing idea for treating the "many-spring" case within our reduced basis framework would be to replace our standard finite element "truth" solver with the adaptive scheme of $[2,3]$ which can treat higher dimensional configuration spaces efficiently.

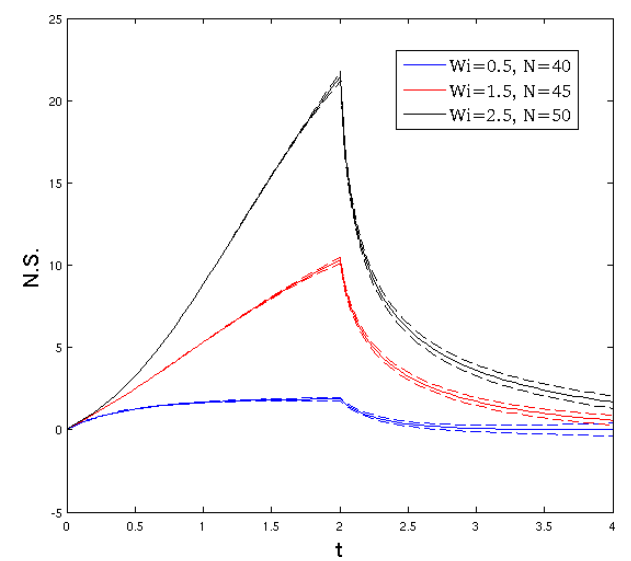

(a)

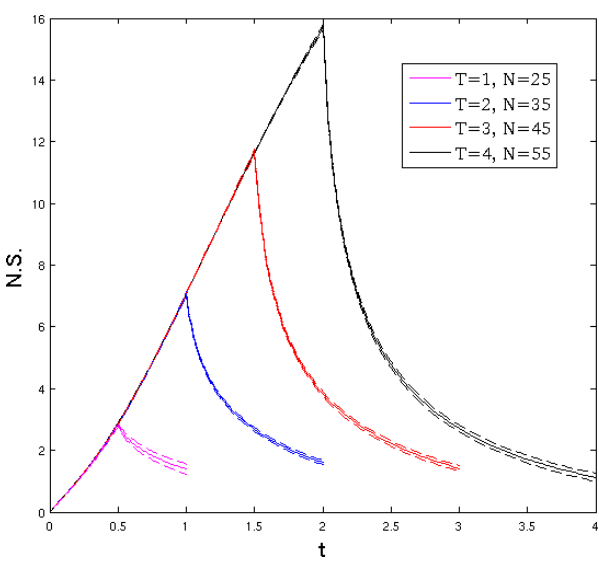

(b)

FIG. 5.5. Plots of the first normal stress difference N.S. as a function of time: N.S.N is plotted as a solid line; N.S. ${ }_{N} \pm \Delta_{N}^{N}$.S. is plotted as dashed lines surrounding N.S..$_{N}$ - the corresponding truth output, N.S. ${ }^{\mathcal{N}}$, is guaranteed to lie between the dashed lines. In (a) we fix $T=4$ and vary $\mathrm{Wi}$ and $N$, and in (b) we fix $\mathrm{Wi}=2$ and vary $T$ and $N$.

Acknowledgments. The authors would like to acknowledge very helpful recent discussions with Professor Gareth McKinley of MIT as well as past fruitful discussions with Sebastien Boyaval and Professor Claude Le Bris of the Ecole Nationale des Ponts et Chaussées and Professor Endre Süli of the University of Oxford. This work was supported by AFOSR Grant FA 9550-07-1-0425.

\section{REFERENCES}

[1] B.O. Almroth, P. Stern, and F.A. Brogan, Automatic choice of global shape functions in structural analysis, AIAA Journal, 16 (1978), pp. 525-528.

[2] A. Ammar, B. Mokdad, F. Chinesta, and R. Keunings, A new family of solvers for some classes of multidimensional partial differential equations encountered in kinetic theory modeling of complex fluids, J. Non-Newtonian Fluid Mech., 139 (2006), pp. 153-176. 


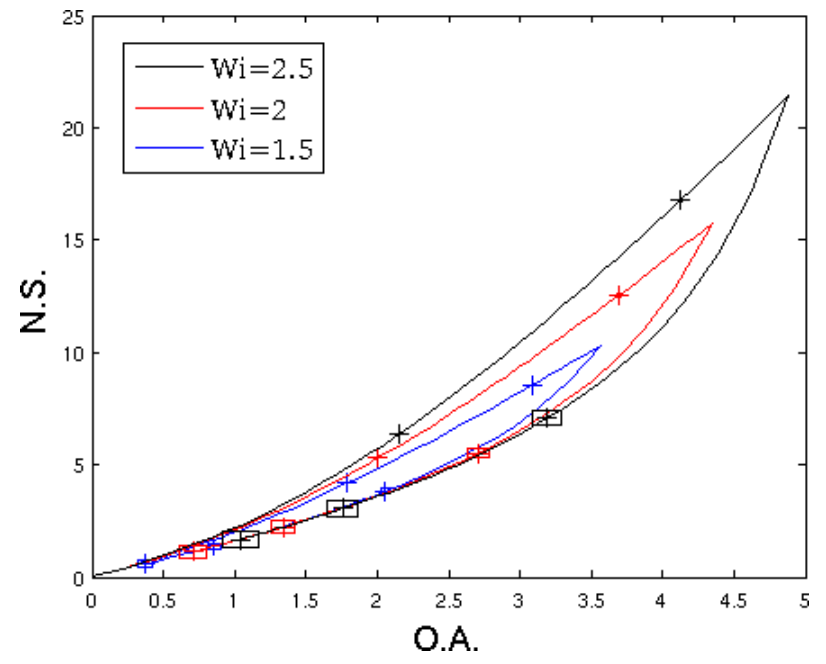

FIG. 5.6. Stress-conformation hysteresis loop for $T=4, N=50$ and $\mathrm{Wi} \in\{1.5,2,2.5\}$. The error boxes centered on ' + ' symbols are placed at time intervals of $t=0.8$; each box defines a region which is guaranteed to contain N.S. ${ }^{\mathcal{N}}$ and O.A. ${ }^{\mathcal{N}}$ at the corresponding time point.

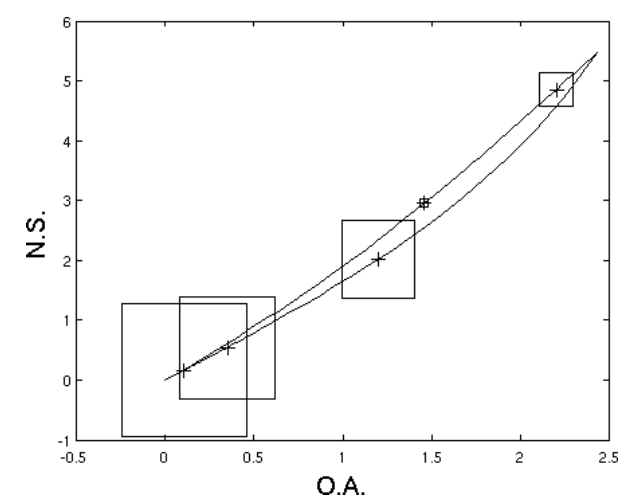

(a)

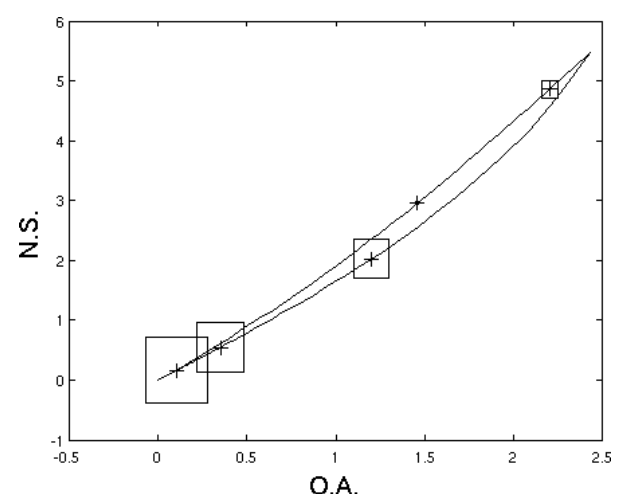

(b)

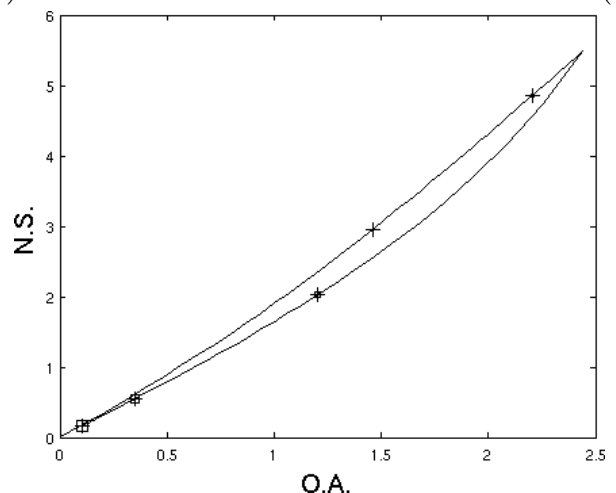

(c)

FIG. 5.7. Stress-conformation hysteresis loops with $\mathrm{Wi}=1, T=4$ and (a) $N=30$, (b) $N=40$, and (c) $N=50$. The error boxes are centered on ' + ' symbols placed at time intervals of $t=0.8$. 
[3] — A new family of solvers for some classes of multidimensional partial differential equations encountered in kinetic theory modelling of complex fluids. Part II: Transient simulation using space-time separated representations, J. Non-Newtonian Fluid Mech., 144 (2007), pp. 98-121.

[4] D. Amsallem, J. Cortial, and C. Farhat, On-demand CFD-based aeroelastic predictions using a database of reduced-order bases and models, in 47th AIAA Aerospace Sciences Meeting, 2009.

[5] D. AmSAllem AND C. FARHAT, Interpolation method for adapting reduced-order models and application to aeroelasticity, AIAA Journal, 46 (2008), pp. 1803-1813.

[6] F. G. Avkhadiev And K.-J. Wirths, Unified Poincaré and Hardy inequalities with sharp constants for convex domains, ZAMM Z. Angew. Math. Mech., 87 (2007), pp. 632-642.

[7] S. Balay, K. Buschelman, V. Eijkhout, W. D. Gropp, D. Kaushik, M. G. Knepley, L. C. McInnes, B. F. Smith, And H. Zhang, PETSc users manual, Tech. Report ANL-95/11 Revision 2.1.5, Argonne National Laboratory, 2004.

[8] R. B. Bird, C. F. Curtiss, R. C. Armstrong, and O. Hassager, Dynamics of Polymeric Liquids, Volume 1, Fluid Mechanics, John Wiley and Sons, second ed., 1987.

[9] —, Dynamics of Polymeric Liquids, Volume 2, Kinetic Theory, John Wiley and Sons, second ed., 1987.

[10] J. Bonvin And M. PICAsso, Variance reduction methods for CONNFFESSIT-like simulations, J. Non-Newtonian Fluid Mech, 84 (1999), pp. 191-215.

[11] S. Boyaval and T. Lelievre, A variance reduction method for parametrized stochastic differential equations using the reduced basis paradigm, arXiv:0906.3600. June 2009.

[12] W. Cazemier, Proper Orthogonal Decomposition and Low Dimensional Models for Turbulent Flows, PhD thesis, University of Groningen, 1997.

[13] C. Chauvière And A. Lozinski, Simulation of complex viscoelastic flows using Fokker-Planck equation: 3D FENE model, J. Non-Newtonian Fluid Mech., 122 (2004), pp. 201-214.

[14] Simulation of dilute polymer solutions using a Fokker-Planck equation, Computers and Fluids, 33 (2004), pp. 687-696.

[15] Y. Chen, J. S. Hesthaven, Y. Maday, and J. Rodriguez, Certified reduced basis methods and output bounds for the harmonic maxwell's equations, SIAM J. Sci. Comput. Submitted.

[16] P. Doyle, E. S. G. Shaqfeh, G. H. McKinley, and S. H. Spiegelberg, Relaxation of dilute polymer solutions following extensional flow, J. Non-Newt. Fluid Mech., 76 (1998), pp. 79110.

[17] J. EfTAng, A parameter domain decomposition approach for reduced basis methods, in ICOSAHOM Proc., 2009. In preparation.

[18] J.P. Fink And W.C. Rheinboldt, On the error behavior of the reduced basis technique for nonlinear finite element approximations, Z. Angew. Math. Mech., 63 (1983), pp. 21-28.

[19] I. Ghosh, Y. L. Joo, G. H. McKinley, R. A. Brown, and R. C. Armstrong, A new model for dilute polymer solutions in flows with strong extensional components, J. Rheol., 45 (2002), pp. 1057-1089.

[20] I. Ghosh, G. H. McKinley, R. A. Brown, and R. C. Armstrong, Defencies of FEnE dumbbell models in describing the rapid stretching of dilute polymer solutions, J. Rheol., 45 (2001), pp. 721-758.

[21] M. D. GunzBurger, Perspectives in Flow Control and Optimization, SIAM, 2003.

[22] B. HaAsdonk And M. OhlBerger, Reduced basis method for finite volume approximations of parametrized linear evolution equations, M2AN Math. Model. Numer. Anal., 42 (2008), pp. 277-302.

[23] C. Helzel and F. Otto, Multiscale simulations of suspensions of rod-like molecules, J. Comp. Phys., 216 (2006), pp. 52-75.

[24] V. Hernandez, J.E. Roman, And V. Vidal, Slepc: A scalable and flexible toolkit for the solution of eigenvalue problems, ACM Trans. Math. Softw., 31 (2005), pp. 351-362.

[25] D.B.P. Huynh, G. Rozza, S. Sen, and A.T. Patera, A successive constraint linear optimization method for lower bounds of parametric coercivity and inf-sup stability constants, Comptes Rendus Mathematique, 345 (2007), pp. 473 - 478.

[26] B. Jourdain, C. Le Bris, And T. Lelievre, On a variance reduction technique for micromacro simulations of polymeric fluids, J. Non-Newtonian Fluid Mech, 122 (2004), pp. 91106.

[27] B. S. Kirk, J. W. Peterson, R. M. Stogner, and G. F. Carey, libMesh: A C++ library for parallel adaptive mesh refinement/coarsening simulations, Engineering with Computers, 23 (2006), pp. 237-254.

[28] D. J. Knezevic And E. SüLI, A heterogeneous alternating-direction method for a micro-macro model of dilute polymeric fluids. Submitted to M2AN, October 2008. 
[29] D. J. KnezeviC ANd E. SÜLI, Spectral galerkin approximation of Fokker-Planck equations with unbounded drift, Accepted to M2AN, October 2008. http://web.comlab.ox.ac.uk/ people/Endre.Suli/biblio.html.

[30] A. Lindner, J. Vermant, And D. Bonn, How to obtain the elongational viscosity of dilute polymer solutions?, Physica A: Statistical Mechanics and its Applications, 319 (2003), pp. 125-133.

[31] A. Lozinski And C. ChauviÈre, A fast solver for Fokker-Planck equation applied to viscoelastic flows calculation: 2D FENE model, Journal of Computational Physics, 189 (2003), pp. 607625.

[32] M.A. Grepl And A.T. Patera, A posteriori error bounds for reduced-basis approximations of parametrized parabolic partial differential equations, Mathematical Modelling and Numerical Analysis, 39 (2005), pp. 157-181.

[33] C. N. Nguyen, G. Rozza, and A. T. Patera, Reduced basis approximation and a posteriori error estimation for the time-dependent viscous burgers equation, Calcolo, (2008).

[34] N. C. Nguyen, G. Rozza, D. B. P. Huynh, and A. T. Patera, Reduced basis approximation and a posteriori error estimation for parametrized parabolic PDEs; application to realtime Bayesian parameter estimation, in Computational Methods for Large Scale Inverse Problems and Uncertainty Quantification, Biegler, Biros, Ghattas, Heinkenschloss, Keyes, Mallick, Tenorio, van Bloemen Waanders, and Willcox, eds., John Wiley and Sons, UK, 2009. (submitted).

[35] A.K. Noor, Recent advances in reduction methods for nonlinear problems, Comput. Struct., 13 (1981), pp. 31-44.

[36] H. C. Öttinger, Stochastic Processes in Polymeric Fluids, Springer, 1996.

[37] R. G. Owens and T. N. Phillips, Computational Rheology, Imperial College Press, 2002.

[38] T. T. Perkins, D. E. Smith, And S. Chu, Single polymer dynamics in an elongational flow, Science, 276 (1997), pp. 2016-2021

[39] T.A. Porsching And M.Y.L. Lee, The reduced-basis method for initial value problems, SIAM Journal of Numerical Analysis, 24 (1987), pp. 1277-1287.

[40] G. Rozza, D. B. P. Huynh, And A. T. Patera, Reduced basis approximation and a posteriori error estimation for affinely parametrized elliptic coercive partial differential equations: application to transport and continuum mechanics, Arch. Comput. Methods Eng., 15 (2008), pp. 229-275.

[41] R. Sizaire, G. Lielens, I. Jaumain, R. Keunings, and V. Legat, On the hysteretic behaviour of dilute polymer solutions in relaxation following extensional flow, J. Non-Newtonian Fluid Mech., 82 (1999), pp. 233-253.

[42] E. Süli And D. MaYers, An Introduction to Numerical Analysis, Cambridge University Press, 2003.

[43] H. R. WARner, Kinetic theory and rheology of dilute suspensions of finitely extendible dumbbells, Ind. Eng. Chem. Fundamentals, (1972), pp. 379-387. 